\title{
Hyperbolic graphs of surface groups
}

\author{
HONGLIN MiN
}

\begin{abstract}
We give a sufficient condition for the fundamental group of a reglued graph of surfaces to be hyperbolic. A reglued graph of surfaces is constructed by cutting a fixed graph of surfaces along the edge surfaces, then regluing by pseudo-Anosov homeomorphisms of the edge surfaces. By carefully choosing the regluing homeomorphism, we construct an example of such a reglued graph of surfaces, whose fundamental group is not abstractly commensurable to any surface-by-free group, ie which is different from all the examples given by Mosher [10].
\end{abstract}

20F67, 20F65; 57M07, 20F28

\section{Introduction}

The fundamental group of the mapping torus of a pseudo-Anosov homeomorphism of an oriented closed hyperbolic surface is hyperbolic. This was first proved by Thurston. A direct proof was given by Bestvina and Feighn [1]. Using their idea, Mosher [10] proved the following theorem.

Consider an oriented closed hyperbolic surface $S$. Let $\Phi_{1}, \ldots, \Phi_{m} \in \operatorname{MCG}(S)$ be an independent set of pseudo-Anosov mapping classes of $S$, and let $\phi_{1}, \ldots, \phi_{m} \in \operatorname{Homeo}(S)$ be pseudo-Anosov representatives of $\Phi_{1}, \ldots, \Phi_{m}$ respectively. If $i_{1}, \ldots, i_{m}$ are large enough positive integers, then the fundamental group of the graph of spaces $\mathcal{G}$, as shown in Figure 1, is a hyperbolic group. In the statement of this theorem, by saying a set $B$ of pseudo-Anosov mapping classes is independent, we mean the sets $\operatorname{Fix}(\Phi)$ are pairwise disjoint for $\Phi \in B$, where $\operatorname{Fix}(\Phi)$ consists of the attractor and the repeller of $\Phi$ on the space of projective measured foliations $\mathcal{P} \mathcal{M F}(S)$.

A graph of surfaces $S \Gamma$ consists of an oriented, connected, finite underlying graph $\Gamma$, a function which assigns to each vertex a closed hyperbolic surface or orbifold, to each edge a closed hyperbolic surface, and another function which assigns to each oriented edge a covering map from the edge surface to the vertex surface of the origin of the edge. In the cases studied in this paper, we change the canonical graph of surfaces by cutting along the edge surfaces, then choosing pseudo-Anosov homeomorphisms of the edge surfaces, precomposing with one of the corresponding attaching maps, 


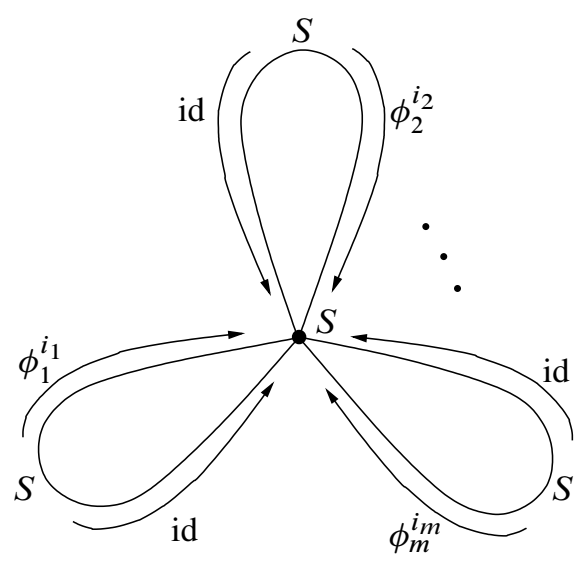

Figure 1

then regluing. We call it a graph of surfaces with pseudo-Anosov regluing. Thus, the mapping torus of a pseudo-Anosov homeomorphism can be considered as this type of space where the underlying graph consists of only one vertex and one edge, and the vertex and edge spaces are the same hyperbolic surface. The case studied by Mosher is a different reglued graph of surfaces where the underlying graph consists of only one vertex and, in addition, the vertex and edge spaces are the same hyperbolic surface $S$.

We shall extend Mosher's theorem to the general graphs of surfaces with pseudo-Anosov regluing. Theorem 1.1 says that if the pseudo-Anosov homeomorphisms are chosen to satisfy an appropriate independence condition, then the fundamental group of the reglued graph of surfaces is word hyperbolic, when these homeomorphisms are replaced with sufficiently high powers of themselves.

We shall describe this cutting and regluing process in more detail. Let $S \Gamma$ be a graph of surfaces with the underlying graph $\Gamma$, let $E$ be the set of oriented edges of $\Gamma$, and let $V$ be the set of vertices of $\Gamma$. For each $e \in E$, let $S_{e}$ be the corresponding edge surface. For each oriented edge $e$, there is a finite covering map $p_{e}: S_{e} \rightarrow F_{o(e)}$, where $F_{o(e)}$ is the vertex surface of the origin $o(e)$ of the edge $e$. For each inverse pair of oriented edges $e, \bar{e}$, there is an inverse pair of homeomorphisms $g_{e}: S_{e} \rightarrow S_{\bar{e}}, g_{e}^{-1}: S_{\bar{e}} \rightarrow S_{e}$. Let $\varphi=\left\{\phi_{e} \mid e \in E\right\}$, where $\phi_{e}: S_{e} \rightarrow S_{e}$ is a pseudo-Anosov homeomorphism of $S_{e}$. Notice that $\phi_{\bar{e}}=\phi_{e}^{-1}$. Also, let $S \Gamma_{\varphi}$ be the graph of surfaces with pseudo-Anosov regluing obtained from $S \Gamma$ by cutting along each $S_{e}$ and regluing using $\phi_{e}$, ie in the reglued graph of surfaces, the effect is to replace the map $g_{e}: S_{e} \rightarrow S_{\bar{e}}$ by the map $g_{e} \circ \phi_{e}$, for $e \in E$. Let $\boldsymbol{m}=\left\{m_{e} \mid e \in E\right\}$, where $m_{e}$ are positive integers, and let $S \Gamma_{\varphi^{m}}$ be the graph of surfaces obtained from $S \Gamma$ by regluing using $\phi_{e}^{m_{e}}$ for each $e \in E$. 
Given a vertex $v$ of the underlying graph $\Gamma$, let $F_{v}$ be the corresponding vertex surface (or orbifold). For each $v \in V$, let $I_{v}=\left\{i \mid e_{i}\right.$ is an oriented edge such that the origin of $e_{i}$ is $\left.v\right\}$. For each $v \in V$ and each $i \in I_{v}$, there is a finite index covering map $p_{i}: S_{i} \rightarrow F_{v}$, where $S_{i}$ is a shorthand notation of $S_{e_{i}}$. If an oriented edge $e_{i}$ has $v$ as both its origin and terminal vertex, the covering maps $p_{i}: S_{i} \rightarrow F_{v}$ and $p_{\bar{i}} \circ g_{i}: S_{i} \rightarrow S_{\bar{i}} \rightarrow F_{v}$ might be different, where $g_{i}$ is a shorthand notation of $g_{e_{i}}$. The portion of $S \Gamma_{\varphi^{m}}$ around a vertex space $F_{v}$ could be as in Figure 2.

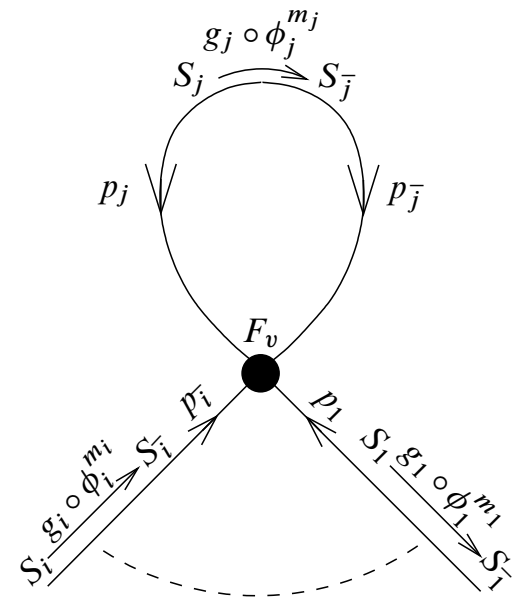

Figure 2

For the purpose of Theorem 1.1, fix a hyperbolic structure on each vertex surface $F_{v}$. For each $v \in V$ and each $i \in I_{v}$, suppose $S_{i}$ is equipped with the metric pulled back by the covering map $p_{i}: S_{i} \rightarrow F_{v}$. Hence, for each covering map $p_{i}$, there is the derivative map D $p_{i}: \mathbf{P} S_{i} \rightarrow \mathbf{P} F_{v}$, where $\mathbf{P} S_{i}$ and $\mathbf{P} F_{v}$ are the projective tangent bundles of $S_{i}$ and $F_{v}$ respectively. For an oriented edge $e_{j}$, let $\phi_{j}^{m_{j}}: S_{j} \rightarrow S_{j}$ be the pseudo-Anosov homeomorphism for the edge $e_{j}$, with the stable geodesic lamination $\Lambda_{j}^{s} \subset S_{j}$. The stable geodesic lamination $\Lambda \frac{s}{\bar{j}} \subset S_{\bar{j}}$ of $\left(\phi_{\bar{j}}^{m_{j}}\right)=g_{j} \phi_{j_{m_{j}}}^{-m_{j}} g_{j}^{-1}$ is homotopic to the image under $g_{j}$ of the unstable geodesic lamination of $\phi_{j}^{m_{j}}$. The geodesic laminations $\Lambda_{j}^{s}$ and $\Lambda_{j}^{s}$ are independent of the choice of the exponent $m_{j}$. In the following, let $\mathrm{T} \Lambda_{i}^{s}$ denote the unit tangent space of $\Lambda_{i}^{s}$.

The Main Theorem of this paper is:

Theorem 1.1 Let $S \Gamma_{\varphi^{m}}$ be a graph of surfaces with pseudo-Anosov regluing. Let $\Gamma$ be its underlying graph. If for each vertex $v \in \Gamma$, and for each $i \in I_{v}$, the derivative maps $\mathrm{D} p_{i} \mid \mathrm{T} \Lambda_{i}^{s}$ are injections and their images are disjoint compact subsets of $\mathbf{P} F_{v}$, then the fundamental group of $S \Gamma_{\boldsymbol{\varphi}^{\boldsymbol{m}}}$ is hyperbolic, when $m_{i} \in \boldsymbol{m}$ are sufficiently large. 
The proof of the hyperbolicity of $S \Gamma_{\varphi^{m}}$ depends ultimately on the Combination Theorem of Bestvina and Feighn [1]. The Combination Theorem says that if the quasi-isometrically embedded condition (which is automatically satisfied in the cases studied in this paper) and the hallways flare condition (which is much more difficult to check) both hold, then $S \Gamma_{\varphi^{m}}$ is a hyperbolic space. In order to check that the hallways flare condition is satisfied, we need to extend the Parallel Corresponds Lemma of Mosher [10], the key in that paper, to a new version of the Parallel Corresponds Lemma. The reason is that Mosher's Parallel Corresponds Lemma only applies to closed geodesics, but we need to study the preimages of closed curves on the vertex surfaces, and these might not continue to be closed curves.

The idea of the proof of Theorem 1.1 is this, the new version of Parallel Corresponds Lemma implies that if a curve is sufficiently far from the stable geodesic lamination $\Lambda_{\phi}^{s}$ of a pseudo-Anosov $\phi$, then it is stretched by $\phi^{m}$ by a definite amount for sufficiently large $m$. The hypothesis of the disjointness of $\mathrm{D} p_{i} \mid \mathrm{T} \Lambda_{i}^{s}$ implies no curve is close to more than one of the $\Lambda_{i}^{s}$. Therefore, the "all but one stretch" condition, similar to Mosher's " $2 m-1$ out of $2 m$ stretch", implies the hyperbolicity of $S \Gamma_{\varphi^{m}}$.

Here are some applications of this theorem.

First: Let $S$ be a closed hyperbolic surface, let $G, H$ be finite subgroups of the mapping class group $\operatorname{MCG}(S)$, and let $\Phi \in \operatorname{MCG}(S)$ be a pseudo-Anosov mapping class. Suppose $G, H$ each have trivial intersection with the virtual centralizer of $\langle\Phi\rangle$ in $\operatorname{MCG}(S)$. Then for sufficiently large $n$, the subgroup $A$ of $\operatorname{MCG}(S)$ generated by $G, \Phi^{n} H \Phi^{-n}$ is isomorphic to the free product of these subgroups. Even more, $A$ is a virtual Schottky subgroup of $\operatorname{MCG}(S)$, in the sense of Farb and Mosher [4].

Second: Let $\mathcal{G}_{\phi^{m}}$ be a graph of surfaces with regluing as in Figure 3, where $S$ and $F$

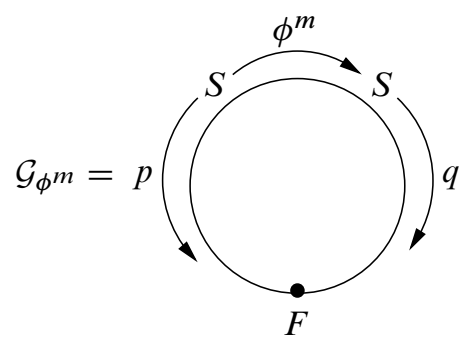

Figure 3

are genus 3 and 2 tori, and $\phi: S \rightarrow S$ is a pseudo-Anosov homeomorphism. Suppose there exist simple closed curves $a \subset F$ and $c \subset S$, as shown in Figure 4, such that $p^{-1}(a)=c, c \subset q^{-1}(a)$, and $q^{-1}(a)$ is disconnected. In addition, suppose that in the 
group $\operatorname{MCG}(S)$, the virtual centralizer of $\langle\Phi\rangle$ has trivial intersection with the deck transformation groups of $p$ and $q$, where $\Phi$ is the mapping class of $\phi$. Then $\pi_{1}\left(\mathcal{G}_{\phi^{m}}\right)$ is hyperbolic when $m$ is sufficiently large.
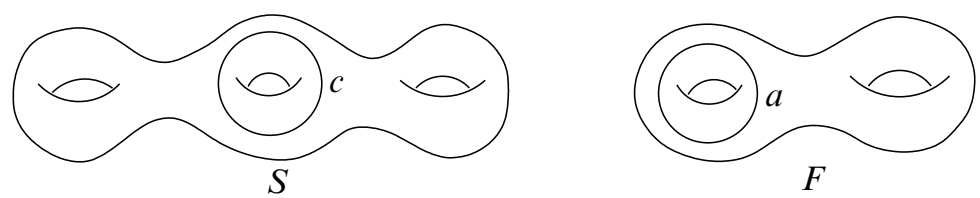

Figure 4

More interestingly, we will see that there exists a pseudo-Anosov homeomorphism $\phi$ of $S$, such that $\pi_{1}\left(\mathcal{G}_{\phi^{m}}\right)$ is not commensurable to $\pi_{1}\left(S^{\prime}\right) \rtimes K$, for any oriented, closed hyperbolic surface $S^{\prime}$, and for any free group $K$, where $\mathcal{G}_{\phi^{m}}$ is as above. Moreover, $\pi_{1}\left(\mathcal{G}_{\phi^{m}}\right)$ is not even quasi-isometric to any surface-by-free group. Therefore $\pi_{1}\left(\mathcal{G}_{\phi^{m}}\right)$ is different from all the hyperbolic groups constructed by Mosher [10].

Problem Do there exist some reducible homeomorphisms of the edge surfaces, such that the graph of surfaces with reducible homeomorphism regluing are hyperbolic?

Problem Is Theorem 1.1 still true when the vertex and edge groups are free groups?

\section{Preliminaries}

In this section, we recall some preliminaries about combinatorial and geometric group theory and some facts about hyperbolic geometry which will be needed later.

Graphs of surfaces Let $\Gamma$ be an oriented, connected, finite graph, let $e$ be an oriented edge of $\Gamma$ and let $\bar{e}$ be the inverse edge of $e$. The vertex $o(e)$ is the origin of $e$ and the vertex $t(e)$ is the terminal of $e$, obviously $o(e)=t(\bar{e})$.

A graph of surfaces $S \Gamma$ consists of an oriented, connected, finite graph $\Gamma$ and a function which assigns to each vertex $v \in \Gamma$ a closed hyperbolic surface or orbifold $F_{v}$, to each pair of oriented edges $e, \bar{e}$ closed hyperbolic surfaces $S_{e}, S_{\bar{e}}$ and an inverse pair of homeomorphisms $g_{e}: S_{e} \rightarrow S_{\bar{e}}, g_{\bar{e}}: S_{\bar{e}} \rightarrow S_{e}$, and to each edge $e$ a continuous map $p_{e}: S_{e} \rightarrow F_{o(e)}$, such that $p_{e}$ induces an injection on the fundamental groups. In most of our cases, the $p_{e}$ are covering maps for every edge $e$ of $\Gamma$.

Given a graph of surfaces $S \Gamma$, we can define the total space $S_{\Gamma}$ as the quotient of the disjoint union $\left(\bigcup\left\{F_{v} \mid v \in V(\Gamma)\right\}\right) \bigcup\left(\bigcup\left\{S_{e} \times I \mid e \in E(\Gamma)\right\}\right)$ by identifying the 
equivalent classes: $(s, 1) \sim p_{e}(s)$ for $(s, 1) \in S_{e} \times I, p_{e}(s) \in F_{t(e)} ;(s, 0) \sim\left(g_{e}(s), 0\right)$ for $(s, 0) \in S_{e} \times I,\left(g_{e}(s), 0\right) \in S_{\bar{e}} \times I$, where $g_{e}: S_{e} \rightarrow S_{\bar{e}}$. The fundamental group of the graph of surfaces $\pi_{1}(S \Gamma)$ is defined to be the fundamental group of the total space $S_{\Gamma}$. There is a projection map $\pi: S_{\Gamma} \rightarrow \Gamma$, such that each vertex surface $F_{v}$ maps to the vertex $v$, and $S_{e} \times I \cup S_{\bar{e}} \times \bar{I} \rightarrow e$, and $\pi$ is a surjection.

The universal cover $\widetilde{S \Gamma}$ of $S \Gamma$ is a union of copies of the universal covers $\widetilde{S_{e}} \times I$ and $\widetilde{F_{v}}$. In $\widetilde{S \Gamma}$, if we identify each copy of $\widetilde{F_{v}}$ to a point and each copy of $\widetilde{S_{e}} \times I$ to a copy of $I$, then we obtain a graph $t$ and there is a canonical projection map $\tilde{\pi}: \widetilde{S \Gamma} \rightarrow t$. It is not hard to see that $t$ is a tree, called the Bass-Serre tree. The action of $\pi_{1}(S \Gamma)$ on $\widetilde{S \Gamma}$ descends to an action of $\pi_{1}(S \Gamma)$ on $t$, where the quotient graph coincides with the original graph $\Gamma$, and the stabilizers of each vertex and each edge of $t$ are conjugates of corresponding fundamental groups of $F_{v}$ and $S_{e}$.

Construction of pseudo-Anosov homeomorphisms In a surface $S, \mathcal{C}$ is an essential curve system, if $\mathcal{C}=\left\{c_{1}, \ldots, c_{n}\right\}$, where $c_{1}, \ldots, c_{n}$ are nontrivial simple closed curves on $S$ which are pairwise disjoint and pairwise nonhomotopic.

Let $\mathcal{C}$ and $\mathcal{D}$ be two disjoint essential curve systems, $\mathcal{C}$ hits $\mathcal{D}$ efficiently if $\mathcal{C}$ intersects $\mathcal{D}$ transversely, and no component on $S \backslash(\mathcal{C} \cup \mathcal{D})$ is a bigon, ie the interior of a disk whose boundary consists of one $\operatorname{arc}$ of $C \in \mathcal{C}$ and one $\operatorname{arc}$ of $D \in \mathcal{D}$. We say that $\mathcal{C} \cup \mathcal{D}$ fills $S$ if the components of the complement of $(\mathcal{C} \cup \mathcal{D})$ are disks.

The following shows how to construct pseudo-Anosov homeomorphisms.

Theorem 2.1 (Penner [13]) Suppose that $\mathcal{C}$ and $\mathcal{D}$ are essential curve systems in an oriented surface $F$ so that $\mathcal{C}$ hits $\mathcal{D}$ efficiently and $\mathcal{C} \cup \mathcal{D}$ fills $F$. Let $R\left(\mathcal{C}^{+}, \mathcal{D}^{-}\right)$ be the free semigroup generated by the Dehn twists $\left\{\tau_{c}^{+}: c \in \mathcal{C}\right\} \cup\left\{\tau_{d}^{-1}: d \in \mathcal{D}\right\}$. Each component map of the isotopy class of $\omega \in R\left(\mathcal{C}^{+}, \mathcal{D}^{-}\right)$is either the identity or pseudo-Anosov, and the isotopy class of $\omega$ is itself pseudo-Anosov if each $\tau_{c}^{+}$and $\tau_{d}^{-1}$ occur at least once in $\omega$.

Surface group extensions A surface group extension is a short exact sequence of the form

$$
1 \rightarrow \pi_{1}(S, x) \rightarrow \Gamma \rightarrow G \rightarrow 1
$$

where $S$ is a closed, oriented surface of genus $g \geq 2$. The canonical example is the Birman exact sequence

$$
1 \longrightarrow \pi_{1}(S, x) \stackrel{i}{\longrightarrow} \operatorname{MCG}(S, x) \stackrel{q}{\longrightarrow} \operatorname{MCG}(S) \longrightarrow 1
$$


where $\operatorname{MCG}(S)$ is the mapping class group of $S, \operatorname{MCG}(S, x)$ is the mapping class group of $S$ punctured at $x$. This short exact sequence is universal for surface group extensions, meaning that for any extension in the short exact sequence (1), there exists a commutative diagram

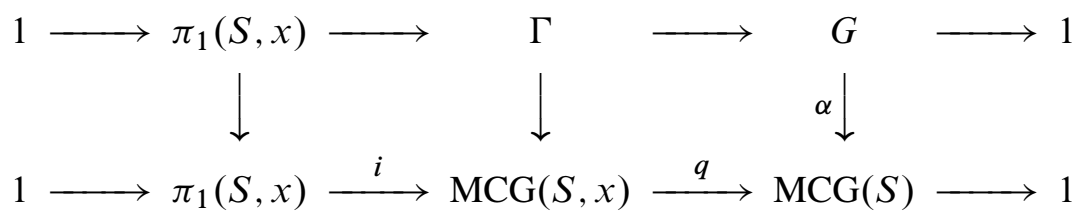

where $\Gamma$ is identified with the pullback group

$$
\Gamma_{\alpha}=\{(\phi, \gamma) \in \operatorname{MCG}(S, x) \times G \mid q(\phi)=\alpha(\gamma)\},
$$

$\alpha$ is a homomorphism from $G$ to $\operatorname{MCG}(S)$, and the homomorphisms $\Gamma \rightarrow G$ and $\Gamma \rightarrow \operatorname{MCG}(S, x)$ are the projection homomorphisms of the pullback group. We are more interested in the case where $\alpha$ is an inclusion.

The virtual centralizer of $\Phi$ Given a subgroup $H$ of a group $G$, the virtual centralizer $\operatorname{VC}(H)$ of $H$ in $G$ is the subgroup of all $g \in G$ which commute with a finite index subgroup of $H$. The virtual centralizer of an infinite cyclic pseudo-Anosov subgroup has a nice geometric description. Let $\mathcal{P M L}(S)$ denote the space of projective measured laminations of the surface $S$. Let $\Lambda^{s}, \Lambda^{u} \subset \mathcal{P} \mathcal{M L}$ be the fixed points of a pseudoAnosov mapping class $\Phi$, and let $\operatorname{Fix}\left\{\Lambda^{s}, \Lambda^{u}\right\}$ denote the subgroup in $\operatorname{MCG}(S)$ whose elements fix $\Lambda^{s}$ and $\Lambda^{u}$ pointwise. Mosher [12] proved that $\operatorname{Fix}\left\{\Lambda^{s}, \Lambda^{u}\right\}=\operatorname{VC}\langle\Phi\rangle$.

Facts of hyperbolic geometry Our proofs make heavy use of the following facts of hyperbolic geometry:

Fact 1 For any $0<\delta<1$, and $D>0$, there exists a number $l(\delta, D)$, such that if $\gamma, \alpha$ are geodesic segments of length at least $l(\delta, D)$, and the end points $x, y$ of $\gamma$ have distance at most $D$ from the end points $x^{\prime}, y^{\prime}$ of $\alpha$ respectively, then there exist subsegments $\gamma^{\prime} \subset \gamma, \alpha^{\prime} \subset \alpha$ of lengths at least $(1-\delta) \operatorname{Length}(\gamma)$ and $(1-\delta) \operatorname{Length}(\alpha)$ respectively, such that the Hausdorff distance between $\gamma^{\prime}$ and $\alpha^{\prime}$ is less than $\delta$.

Roughly speaking, for any two geodesic segments, if their end points have bounded distances from each other, then large subsegments of them are arbitrarily close to each other provided the segments are long enough.

Fact 2 Given $k \geq 1, c \geq 0$, there exists a constant $N_{0}(k, c)$, such that any $(k, c)$ quasigeodesic line or segment in hyperbolic space $\mathbb{H}^{2}$ has Hausdorff distance at most $N_{0}(k, c)$ from a geodesic line or segment with the same end points. 
Fact 3 Let $\Lambda_{1}$ and $\Lambda_{2}$ be two minimal geodesic laminations filling a hyperbolic surface $S$. If their lifts $\widetilde{\Lambda}_{1}$ and $\tilde{\Lambda}_{2}$ to the universal cover of $\tilde{S}$ have at least one end point in common, then $\Lambda_{1}=\Lambda_{2}$.

A geodesic lamination $\Lambda$ is minimal if every leaf $L$ is dense, that is, $\bar{L}=\Lambda$. A geodesic lamination $\Lambda \subset S$ is a filling lamination if no simple closed curve in $S$ is disjoint from $\Lambda$.

The reason this fact is true is that two minimal filling surface geodesic laminations either transversely intersect each other or are equal to each other.

From Fathi, Laudenbach and Poenaru [6], we know that the stable and unstable geodesic laminations of a pseudo-Anosov homeomorphism are minimal and filling.

\section{The Main Theorem}

We will give a new version of the Parallel Corresponds Lemma and use it to prove Theorem 1.1. Moreover we will reformulate the hypothesis of Theorem 1.1. The original Corresponds Lemma was given by Mosher [10].

\subsection{All but one stretch}

Mosher proved the hyperbolicity of $\pi_{1}(\mathcal{G})$ using a lemma called " $2 m-1$ out of $2 m$ stretch", where $\mathcal{G}$ as shown in Figure 1 . The " $2 m-1$ out of $2 m$ stretch" Lemma says that for any $\lambda>1$, if $i_{1}, \ldots, i_{m}$ are sufficiently large integers, then for any nontrivial element $g \in \pi_{1}(S, x)$, at least $2 m-1$ out of the $2 m$ elements $\left\{\phi_{1}^{i_{1}}, \phi_{1}^{-i_{1}}, \ldots, \phi_{m}^{i_{m}}, \phi_{m}^{-i_{m}}\right\}$ stretch $g$ by a factor of $\lambda$. In this lemma, $\phi_{1}, \ldots, \phi_{m}$ are considered as elements in $\operatorname{MCG}(S, x)$, since by taking powers, we can assume $\phi_{1}, \ldots, \phi_{m}$ each have a fixed point $x$. We use the same symbol $\phi$ to denote the corresponding element of $\operatorname{Aut}\left(\pi_{1}(S, x)\right)$, for any $\phi \in \operatorname{MCG}(S, x)$. $\phi$ stretches $g$ by a factor of $\lambda$ if the length of $\phi(g)$ is greater than $\lambda$ times the length of $g$, where the length is the word metric length.

Following Mosher's idea, we shall prove the hyperbolicity of $S \Gamma_{\varphi^{m}}$ by showing that it satisfies the "all but one stretch" Condition. We say that $S \Gamma_{\varphi^{m}}$ satisfies the "all but one stretch" Condition, if there exist $\lambda>1$ and $C>0$, such that for any based geodesic segment $\gamma$ on a vertex surface $F_{v}$ of $S \Gamma_{\varphi^{m}}$, with length at least $C$, all but at most one of the preimages of $\gamma$ are stretched by corresponding $\phi_{i}^{m_{i}}$ by a factor of at least $\lambda$ for any $i \in I_{v}$. The proof of the Main Theorem shows that $S \Gamma_{\varphi^{m}}$ satisfies this condition. 


\subsection{A new version of the Parallel Corresponds Lemma}

Consider a pseudo-Anosov mapping class $\Phi \subset \operatorname{MCG}(S)$ and let $\phi \in \operatorname{Homeo}(S)$ be a pseudo-Anosov representative with the stable and unstable measured foliations $f_{\phi}^{s}, f_{\phi}^{u}$. Recall that the transverse measures on $f_{\phi}^{s}$ and $f_{\phi}^{u}$ define a singular Euclidean structure on $S$, with isolated cone singularities. We call the leaves of $f_{\phi}^{s}$ the horizontal leaves and the leaves of $f_{\phi}^{u}$ the vertical leaves. The singular Euclidean structure determines a metric $d_{\phi}$ on $S$ in which each path can be homotoped rel end points to a unique geodesic. The lifts to the universal covers of the hyperbolic metric and the singular Euclidean metric are quasi-isometric.

In the following, for a homotopy class $\gamma$ of a curve rel end points, let $\gamma^{h}$ denote the hyperbolic geodesic segment in the homotopy class of $\gamma$, and let $\gamma^{E}$ denote the singular Euclidean geodesic segment in the same homotopy class. For a homotopy class $\gamma$, let $|\gamma|$ denote the hyperbolic length of $\gamma^{h}$, let $|\gamma|_{E}$ denotes the singular Euclidean length of $\gamma^{E}$.

Given $0<\eta<1$, define slope ${ }_{\phi}^{\eta}$ to be the set of all homotopy classes $\gamma$, such that the (unsigned) Euclidean angle between $\gamma^{E}$ and $f_{\phi}^{s}$ is at least $\eta$, on a subset of $\gamma^{E}$ of length at least $\eta \cdot|\gamma|_{E}$. Given $\lambda>1$, let $\operatorname{stretch}_{\phi}^{\lambda}=\{\gamma||(\phi(\gamma))|>\lambda| \gamma \mid\}$. Let $n$ be a large enough integer, such that if the vector $v \in \mathbb{E}^{2}$ makes an angle of at least $\eta$ with the horizontal axis, then the matrix

$$
\left(\begin{array}{cc}
\lambda_{\phi}^{-n} & 0 \\
0 & \lambda_{\phi}^{n}
\end{array}\right)
$$

stretches $v$ by a factor of at least $\lambda / \eta$, where $\lambda_{\phi}=\lim _{i \rightarrow \infty}\left|\phi^{i}(\alpha)\right|^{1 / i}$ is the stretching factor of $\phi, \alpha$ is a simple closed geodesic on $S$. Since the singular Euclidean metric is quasi-isometric to the hyperbolic metric, it follows that given $\phi, 0<\eta<1$ and $\lambda>1$, there exists $N$ such that if $n \geq N$, then slope ${ }_{\phi}^{\eta} \subset \operatorname{stretch}_{\phi^{n}}^{\lambda}$.

An $\eta$-lever is a homotopy from a singular Euclidean geodesic segment $\alpha$ to a horizontal segment $\beta$, where $\beta$ is a segment of a nonsingular leaf of the horizontal foliation $f_{\phi}^{s}$, such that each track of the homotopy is a vertical geodesic segment, possibly degenerate, and each point of $\operatorname{int}(\alpha)$ is disjoint from singularities during the homotopy, and $\operatorname{int}(\alpha)$ makes an angle of at most $\eta$ with the horizontal leaves. In Mosher [10], $\beta$ is not necessarily a segment of a nonsingular leaf. But we can always assume $\beta$ is a segment of a nonsingular leaf, because there exist nonsingular leafs which are arbitrary close to a singular leaf. Notice that the angle between a singular Euclidean geodesic and the horizontal leaves changes only when the singular Euclidean geodesic passes through a singularity. Therefore, the interior of $\alpha$ has a constant angle with the horizontal leaf. 
A lever is denoted by $(\alpha, \beta)$, where $\alpha$ is called the inclined edge of the lever, and $\beta$ is called the horizontal edge of the lever. A lever is maximal if and only if a singularity is contained in the track of each end point of $\alpha$. The length of the lever is $|\alpha|_{E}$, the height of the lever is the maximum singular Euclidean length of the tracks of the points of $\alpha$, which is achieved at the endpoints.

Proposition 3.1 (Mosher [10]) For any $l, H>0$, there exists $\eta(l, H)>0$, so that every maximal $\eta$-lever has length at least $l$ and height at most $H$.

The proof is given in the first seven paragraphs of the proof of the sublemma on page 3451 of Mosher [10]. This proposition will be used in the proof of the following lemma.

In the proof of the following lemma, we need some facts. It is well known that the measured foliations $f_{\phi}^{s}, f_{\phi}^{u}$ can be straightened to measured geodesic laminations $l_{\phi}^{s}, l_{\phi}^{u}$. Actually, there is a 1-1 correspondence between leaves of $l_{\phi}^{s}$ and smooth leaves of $f_{\phi}^{s}$, where a smooth leaf is either a nonsingular leaf or the union of two singular half-leaves meeting at a singularity with angle $180^{\circ}$. Similarly for $f_{\phi}^{u}$. The singularities are discrete, so the length of any geodesic connecting them has a positive lower bound.

Lemma 3.2 (A new version of Parallel Corresponds Lemma) Given any pseudoAnosov homeomorphism $\phi$ and $0<\epsilon<1$, there exist $0<\eta<1$ and $L>0$ such that for any homotopy class $\gamma$, if $\gamma \notin$ slope $_{\phi}^{\eta}$ and $|\gamma|_{E} \geq L$, then on a subset of $\gamma^{h}$ of length at least $(1-\epsilon)|\gamma|$, the distance between the tangent line of $\gamma^{h}$ and the set $l_{\phi}^{s}$, measured in $\mathbf{P} S$, is at most $\epsilon$.

The differences between the Parallel Corresponds Lemma of Mosher [10] and this new version are as follows. In [10], the Parallel Corresponds Lemma works only for closed based geodesics, and the word metric is used to define the stretching factor; in this paper, the new version of the Parallel Corresponds Lemma works for nonclosed geodesics as well, and the hyperbolic metric is used to define the stretching factor.

Proof The first step is to find long subsegments $\alpha_{i} \subset \gamma^{E}$ and segments $\beta_{i}$ of leaves of $f_{\phi}^{s}$, such that $\alpha_{i}$ is homotopic to $\beta_{i}$. Then we shall project $\alpha_{i}$ to a subsegment of $\gamma^{h}$ and project $\beta_{i}$ to a segment of a leaf $B_{i}^{h}$ of $l_{\phi}^{s}$, and show that the long subsegments of these projections are very close to each other. Finally we shall prove the long subsegments of $\gamma^{h}$ are almost covered by the long subsegments of these projections.

For $\gamma \notin \operatorname{slope}_{\phi}^{\eta}$, let $\left\{\left(\alpha_{i}, \beta_{i}\right)\right\}$ be the set of all maximal $\eta$-levers of $\gamma^{E}$, where the inclined edge $\alpha_{i}$ is a subsegment of $\gamma^{E}$ and the horizontal edge $\beta_{i}$ is a segment of some nonsingular leaf $B_{i}^{E}$ of $f_{\phi}^{s}$. 
Step 1 First, let $H=1$. By Proposition 3.1, we know that for any $l>0$, there exists $\eta>0$ such that every maximal $\eta$-lever $\left\{\left(\alpha_{i}, \beta_{i}\right)\right\}$ has length at least $l$ and height at most $H=1$. The first step is proven.

Step 2 we shall construct long subsegments of $\gamma^{h}$ from the inclined edges $\alpha_{i} \subset \gamma^{E}$ of the maximal levers, such that these long subsegments of $\gamma^{h}$ have small distance from $l_{\phi}^{s}$ measured in $\mathbf{P} S$. For the remainder of the proof, the distance and length mean hyperbolic distance and length, unless we will use the terminology Euclidean distance and length.

We know that any nonsingular leaf $B_{i}^{E}$ of $f_{\phi}^{s}$ is a quasigeodesic under the hyperbolic metric, and it can be straightened to a unique leaf $B_{i}^{h}$ of $l_{\phi}^{s}$. Denote by $\delta_{i} \subset \gamma^{h}$ and $\sigma_{i} \subset B_{i}^{h}$ the closest point projections from $\alpha_{i} \subset \gamma^{E}$ to $\gamma^{h}$ and from $\beta_{i} \subset B_{i}^{E}$ to $B_{i}^{h}$, respectively. We shall see that a long subsegment of $\delta_{i}$ has small distance from $\sigma_{i}$, for all $i$.

Since $\gamma^{E}$ is a $(k, c)$ quasigeodesic segment contained in the $N_{0}(k, c)$ neighborhood of $\gamma^{h}$, and $\delta_{i}, \alpha_{i}$ are subsegments of $\gamma^{h}, \gamma^{E}$ respectively, it follows that the distances between the end points of $\delta_{i}$ and $\alpha_{i}$ are not greater than $N_{0}$. For the same reason, the distance between the end points of $\sigma_{i}$ and $\beta_{i}$ are not greater than $N_{0}$. The singular Euclidean distance between the end points of $\beta_{i}$ and $\alpha_{i}$ is less than the height $H=1$. The hyperbolic distances between their end point are at most $m k$, for some $m>0$, because the singular Euclidean and hyperbolic metric are $(k, c)$ quasi-isometric to each other. Therefore the distances between the end points of $\delta_{i}$ and $\sigma_{i}$ are less than $2 N_{0}+m k$. According to Fact 1 , for any $\epsilon_{1}>0$, there exists a constant $L_{1}$ depending on $2 N_{0}+m k$ and $\epsilon_{1}$, so that if the length of $\delta_{i}$ is greater than $L_{1}$, then more than $\left(1-\epsilon_{1}\right)\left|\delta_{i}\right|$ portion of $\delta_{i}$ has distance less than $\epsilon_{1}$ with $\sigma_{i}$.

The condition that the length of $\delta_{i}$ be greater than $L_{1}$ is easy to satisfy. Since $\alpha_{i}$ is a quasigeodesic segment whose end points have distances less than $N_{0}$ from the end points of $\delta_{i}$, there exists a constant $l_{1}>0$, such that if the Euclidean length of $\alpha_{i}$ is greater that $l_{1}$, then the length of $\delta_{i}$ is greater than $L_{1}$. By applying Step 1, we may now choose $\eta$ small enough, so that the Euclidean length of $\alpha_{i}$ is greater than $l_{1}$ for any $i$. Therefore, more than a $\left(1-\epsilon_{1}\right)\left|\delta_{i}\right|$ portion of $\delta_{i}$ has distance less than $\epsilon_{1}$ from $\sigma_{i}$.

So far, we have proved that for any $\epsilon_{1}$, there exists $\eta$, such that if $\gamma \notin \operatorname{slope}_{\phi}^{\eta}$, then we can locate long subsegments $\delta_{i}$ of $\gamma^{h}$, such that more than $\left(1-\epsilon_{1}\right)$ of the length of $\delta_{i}$ has distance less than $\epsilon_{1}$ with $\sigma_{i} \subset B_{i}^{h}$, for any $i$.

Step 3 we will prove that a $\left(1-\epsilon_{1}\right) \sum_{i}\left|\delta_{i}\right|$ portion of $\bigcup_{i}\left(\delta_{i}\right)$ covers long subsegments of $\gamma^{h}$. We call this $\left(1-\epsilon_{1}\right) \sum_{i}\left|\delta_{i}\right|$ part of $\bigcup_{i}\left(\delta_{i}\right)$ the "good" part of $\gamma^{h}$. 
Since $\gamma \notin$ slope $_{\phi}^{\eta}$, on a subset of $\gamma^{E}$ of length at least $(1-\eta)|\gamma|_{E}$, the angle between $\gamma^{E}$ and $f_{\phi}^{s}$ is less than $\eta$, ie the $\eta$-levers cover more than a $(1-\eta)$ portion of $\gamma^{E}$. The worst situation is that the two end subsegments of $\gamma^{E}$ are covered by $\eta$-levers with lengths less than $l_{1}$. In this case, after straightening, the end subsegments of $\gamma^{h}$ may not have distances less than $\epsilon_{1}$ from $B^{h}$. We will only prove this lemma for the worst situation, ie when more than a $\left(1-\epsilon_{1}\right)|\gamma|_{E}$ portion of $\gamma^{E}$ is covered by the union of the maximal $\eta$-levers $\left(\alpha_{i}, \beta_{i}\right)$ and two end $\eta$-levers which cover the two end segments of $\gamma^{E}$ respectively and with lengths less than $l_{1}$.

In the following, the quasi-isometries will be replaced by bi-Lipschitz maps when we are dealing with long segments. Keep in mind that in the following, $\delta_{i}$ is not the projection of an end subsegment of $\gamma^{E}$.

$$
\begin{aligned}
\left(1-\epsilon_{1}\right) \sum_{i}\left|\delta_{i}\right| & \geq\left(1-\epsilon_{1}\right)\left(\sum_{i}\left(\left|\alpha_{i}\right|-2 N_{0}\right)\right) \\
& \geq\left(1-\epsilon_{1}\right)\left(\sum_{i}\left(\frac{\left|\alpha_{i}\right| E}{k}-2 N_{0}\right)\right) .
\end{aligned}
$$

According to Proposition 3.1, we can take $\eta$ to be small enough, so that $\left|\alpha_{i}\right|_{E} \geq l_{2}=$ $4 k N_{0}$ for any $i$, thus

$$
\left(1-\epsilon_{1}\right) \sum_{i}\left|\delta_{i}\right| \geq\left(1-\epsilon_{1}\right) \frac{\sum_{i}\left|\alpha_{i}\right|_{E}}{2 k} .
$$

Since the union of the maximal $\eta$-levers and the two end $\eta$-levers, covers more than $\left(1-\epsilon_{1}\right)|\gamma|_{E}$ portion of $\gamma^{E}$, and we can suppose that the two end $\eta$-levers have lengths less than $l_{1}$,

$$
\left(1-\epsilon_{1}\right) \sum_{i}\left|\delta_{i}\right| \geq\left(1-\epsilon_{1}\right) \frac{\left(1-\epsilon_{1}\right)|\gamma|_{E}-2 l_{1}}{2 k},
$$

taking $|\gamma|_{E}$ to be long enough, so that $|\gamma|_{E} \geq L_{2}=2 l_{1} / \epsilon_{1}$,

$$
\begin{aligned}
\left(1-\epsilon_{1}\right) \sum_{i}\left|\delta_{i}\right| & \geq\left(1-\epsilon_{1}\right) \frac{\left(1-2 \epsilon_{1}\right)|\gamma|_{E}}{2 k} \\
& \geq \frac{\left(1-2 \epsilon_{1}\right)^{2}|\gamma|_{E}}{2 k} .
\end{aligned}
$$

Hence,

$$
\left(1-\epsilon_{1}\right) \sum_{i}\left|\delta_{i}\right| \geq \frac{\left(1-2 \epsilon_{1}\right)^{2}|\gamma|_{E}}{2 k} .
$$


The "bad" parts of $\gamma^{h}$ are of three kinds. The first kind of bad part are the two end subsegments of $\gamma^{h}$ which have lengths less than $L_{1}$. The sum of the lengths of the end subsegments of $\gamma^{h}$ is at most $2 L_{1}$. We can take $|\gamma|_{E}$ to be big enough so that $2 L_{1} \leq \epsilon_{1}|\gamma|_{E}$.

The second kind of bad part of $\gamma^{h}$ is the $\epsilon_{1}\left|\delta_{i}\right|$ portion of $\delta_{i}$ 's which may be outside of the $\epsilon_{1}$ neighborhood of $\sigma_{i}$. Since the projection map cannot prolong length, and the distances between the ends of $\alpha_{i}$ and $\delta_{i}$ are not greater than $N_{0}$,

$$
\sum_{i} \epsilon_{1}\left|\delta_{i}\right| \leq \epsilon_{1} \sum_{i}\left(\left|\alpha_{i}\right|+2 N_{0}\right)
$$

We can take $\eta$ to be small enough, so that $\left|\alpha_{i}\right|_{E} \geq l_{2}=4 k N_{0}$ for any $i$. The singular Euclidean metric and the hyperbolic metric are $k$ bi-Lipschitz; thus, $\left|\alpha_{i}\right|_{E} \leq k\left|\alpha_{i}\right|$. Therefore, $2 N_{0} \leq 2 k N_{0} \leq\left|\alpha_{i}\right| / 2$, thus

$$
\begin{aligned}
\sum_{i} \epsilon_{1}\left|\delta_{i}\right| & \leq \epsilon_{1} \frac{3}{2} \sum_{i}\left|\alpha_{i}\right| \\
& \leq \epsilon_{1} \frac{3}{2} k \sum_{i}\left|\alpha_{i}\right|_{E} \\
& \leq \epsilon_{1} 2 k|\gamma|_{E} .
\end{aligned}
$$

A third kind of bad part of $\gamma^{h}$ is the projection of an $\epsilon_{1}|\gamma|_{E}$ portion of $\gamma^{E}$ which has slope greater than $\epsilon_{1}$ with $f_{\phi}^{s}$. Let $\xi_{i}$ denote this kind of subsegment of $\gamma^{E}$. There is a lower bound $b$ of the Euclidean lengths of $\xi_{i}$ for all $i$, and it equals the minimum of the Euclidean distances between singularities. The sum of the lengths of the projections from $\xi_{i}$ to $\gamma^{h}$ is at most $\sum_{i}\left(k\left|\xi_{i}\right|_{E}+c\right) \leq \sum_{i}\left(k\left|\xi_{i}\right|_{E}+(n-1) k b\right) \leq$ $n \sum_{i}\left(k\left|\xi_{i}\right|_{E}\right) \leq n k \epsilon_{1}|\gamma|_{E}$, for some $n$ satisfying $c \leq(n-1) k b$.

Therefore, the length of the "bad" part of $\gamma^{h}$ is at most the sum of the above three kinds, which is $(2 k+1+n k) \epsilon_{1}|\gamma|_{E}$. Hence, the ratio of the "good" part of $\gamma^{h}$ to the "bad" part of $\gamma^{h}$ is at least $\left(1-2 \epsilon_{1}\right)^{2} /\left(2 k(1+2 k+n k) \epsilon_{1}\right)$. From this, it is easy to see, for any constant $\epsilon$ there exists a small enough $\epsilon_{1}$, such that the ratio of the 'good' part of $\gamma^{h}$ to $\gamma^{h}$ is at least $(1-\epsilon)$.

To recap: for any $\epsilon>0$, we can choose $\epsilon_{1}$ small enough so $\left(1-2 \epsilon_{1}\right)^{2} / 2 k(1+2 k+n k) \epsilon_{1}$ is greater than $1-\epsilon$; therefore, the "good" part of $\gamma^{h}$ covers more than $(1-\epsilon)$ of the total length of $\gamma^{h}$. Next choose $\eta$ small enough so that if $\gamma^{E} \notin$ slope $_{\phi}^{\eta}$, then more than $(1-\epsilon)\left|\delta_{i}\right|$ portion of $\delta_{i}$ has distance less than $\epsilon_{1}$ from $\sigma_{i}$. In addition, take $|\gamma|_{E}$ to be at least $L$, where $L=\max \left\{L_{2}, 2 L_{1} / \epsilon_{1}\right\}$. Hence, if $\eta$ is small enough, $\gamma \notin \operatorname{slope}_{\phi}^{\eta}$ and $|\gamma|_{E} \geq L$, then the large subsegment of $\gamma^{h}$ has distance at most $\epsilon$ to $l_{\phi}^{s}$, measured in $\mathbf{P} S$. 
Given a geodesic lamination $\Lambda$ and $0<\epsilon<1$, let $\mathrm{WN}_{\epsilon}(\Lambda)$ denote the set of all the homotopy classes, $\gamma$, so that on a subset of $\gamma^{h}$ of length at least $(1-\epsilon)|\gamma|$, the distance from the tangent line of $\gamma^{h}$ to the set $\Lambda$, measured in $\mathbf{P} S$, is at most $\epsilon$. Using this notation, the Parallel Corresponds Lemma says that for any $0<\epsilon<1$, there exists $0<\eta<1$ and $L>0$, such that if $\gamma \notin \operatorname{slope}_{\phi}^{\eta}$ and $|\gamma|_{E} \geq L$, then $\gamma \in \operatorname{WN}_{\epsilon}\left(\Lambda^{s}\right)$, where $\Lambda^{s}$ is the measured stable geodesic lamination of $\phi$.

\subsection{Proof of the Main Theorem}

Proof of Theorem 1.1 We shall prove the "all but one stretch" Condition is satisfied, ie there exist $\lambda>1$ and $C>0$, so that for any vertex $w \in \Gamma$, if a based geodesic segment $\gamma_{w}^{h} \subset F_{w}$ has length at least $C$, then all but at most one preimages belong to stretch $_{\phi_{i}}^{\lambda m_{i}}$ for corresponding $\phi_{i}$, for any $i \in I_{w}$, where $I_{w}=\left\{i \mid e_{i}\right.$ is an oriented edge such that the origin of $e_{i}$ is $\left.w\right\}$. Therefore, $S \Gamma_{\varphi^{m}}$ is a hyperbolic surface.

Let $v$ be a vertex of $\Gamma$, let $\gamma_{v}^{h} \subset F_{v}$ be a based geodesic segment. Consider the set $\Sigma=\bigcup_{i \in I_{v}} p_{i}^{-1}\left(\gamma_{v}^{h}\right)$, where $p_{i}^{-1}\left(\gamma_{v}^{h}\right)$ is the set of all preimages of $\gamma_{v}^{h}$ under the map $p_{i}$. Notice that all the elements of $\Sigma$ are based geodesics, since the edge surfaces of $S \Gamma_{\varphi^{m}}$ are equipped with the pullback metrics.

First, we claim that there exist $0<\epsilon_{0}<1$ and $H_{0}>0$, such that if the length of $\gamma_{v}^{h}$ is greater than $H_{0}$, then at most one of the elements of $\Sigma$, say $\beta \in p_{i_{0}}^{-1}\left(\gamma_{v}^{h}\right)$, satisfies $\beta \in \mathrm{WN}_{\epsilon_{0}}\left(\Lambda_{i_{0}}^{s}\right)$, for some $i_{0} \in I_{v}$; all other elements of $\Sigma$ are not contained in $\mathrm{WN}_{\epsilon}\left(\Lambda_{i}^{s}\right)$ for the corresponding $\Lambda_{i}^{s}$. Second, according to Lemma 3.2, for this $\epsilon_{0}$, there exist $0<\eta\left(\epsilon_{0}\right)<1$ and $L\left(\epsilon_{0}\right)>0$, such that for any $\alpha \in \Sigma$ with length $|\alpha|=\left|\gamma_{v}^{h}\right|$ greater than $L\left(\epsilon_{0}\right)$, if $\alpha \notin \mathrm{WN}_{\epsilon_{0}}\left(\Lambda_{j}^{s}\right)$, then $\alpha \in \operatorname{slope}_{\phi_{j}}^{\eta\left(\epsilon_{0}\right)} \subset \operatorname{stretch}_{\phi_{j}}^{\lambda m_{j}}$ for sufficiently large $m_{j}$. So, for any $\gamma_{v}^{h}$ with length greater $C=\max \left\{H, L\left(\epsilon_{0}\right)\right\}$, all but at most one of the preimages of $\gamma_{v}^{h}$ belongs to stretch $\hat{\phi}_{i}^{\lambda_{i}}$ for the corresponding $\phi_{i}$.

Suppose the claim is not true. In other words, for any $\epsilon_{n} \rightarrow 0$, and any $H_{n} \rightarrow \infty$, there exist based geodesic segments $\gamma_{n}^{h} \subset F_{v}$ with lengths at least $H_{n}$ and by passing to a subsequence, without loss of generality, we can suppose $A_{n}^{h} \in p_{1}^{-1}\left(\gamma_{n}^{h}\right)$ and $B_{n}^{h} \in p_{2}^{-1}\left(\gamma_{n}^{h}\right)$ such that $A_{n}^{h} \in \mathrm{WN}_{\epsilon_{n}}\left(\Lambda_{1}^{s}\right)$ and $B_{n}^{h} \in \mathrm{WN}_{\epsilon_{n}}\left(\Lambda_{2}^{s}\right)$. Projecting $A_{n}^{h}$ and $B_{n}^{h}$ to $\Lambda_{1}^{s}$ and $\Lambda_{2}^{s}$ respectively, we see that there exist long subsegments $v_{n} \subset \Lambda_{1}^{s}$ and $\omega_{n} \subset \Lambda_{2}^{s}$, such that $\left|v_{n}\right|,\left|\omega_{n}\right| \rightarrow \infty$, and the distance between $\mathrm{D} p_{1} \mid T v_{n}$ and $\mathrm{D} p_{2} \mid T \omega_{n}$ converges to zero. This contradicts with the fact that $\mathrm{D} p_{1} \mid \mathrm{T} \Lambda_{1}^{s}$ and $\mathrm{D} p_{2} \mid \mathrm{T} \Lambda_{2}^{s}$ are disjoint.

\subsection{Reformulation of Theorem 1.1}

Notation here is the same as in the Introduction. The only difference is that here, the edge surfaces are not necessary equipped with the pullback metrics. 
Let $v$ be a vertex of $\Gamma$, let $y$ be the base point of $F_{v}$, and let $I_{v}$ be as defined before. Consider the set $p_{i}^{-1}(y) \subset S_{i}$ of all the points of $S_{i}$ that cover $y$ via the map $p_{i}$, for $i \in I_{v}$. Let $X=\bigcup_{i \in I_{v}} p_{i}^{-1}(y)$.

Suppose $a \in p_{i}^{-1}(y)$, choose a lift $\tilde{p}_{a}:\left(\widetilde{S}_{i}, \tilde{a}\right) \rightarrow\left(\widetilde{F}_{v}, \tilde{y}\right)$, where $\widetilde{S}_{i}$ and $\widetilde{F}_{v}$ are the universal covers of $S_{i}$ and $F_{v}$ respectively. Let $\Lambda_{i}^{s} \subset S_{i}$ be the stable lamination of $\phi_{i}$ and let $\widetilde{\Lambda}_{i}^{s} \subset \widetilde{S}_{i}$ be the lift of $\Lambda_{i}^{s}$. Notice that $\partial \widetilde{p}_{a}\left(\widetilde{\Lambda}_{i}^{s}\right) \subset \partial \widetilde{F}_{v}$ is well defined independent of the choice of $\tilde{y}$ and $\tilde{a}$. If for any $a \neq b \in X, \partial \widetilde{p}_{a}\left(\tilde{\Lambda}_{i}^{s}\right) \cap \partial \widetilde{p}_{b}\left(\widetilde{\Lambda}_{j}^{s}\right)=\varnothing$, where $a \in p_{i}^{-1}(y), b \in p_{j}^{-1}(y)$, then we say $v$ satisfies the disjointness condition. We only require $a \neq b$, but $i$ might equal $j$. The reformulation of Theorem 1.1 is the following.

Theorem 3.3 Let $S \Gamma_{\varphi^{m}}$ be a finite graph of surfaces with underlying graph $\Gamma$. If for any vertex $v \in \Gamma$, the disjointness condition is satisfied, then $\pi_{1}\left(S \Gamma_{\varphi^{m}}\right)$ is a hyperbolic group, when the $m_{i} \in \boldsymbol{m}$ are sufficiently large.

We shall show the equivalence of the hypotheses of Theorem 1.1 and Theorem 3.3.

First, suppose $\mathrm{D} p_{i}\left(\mathrm{~T} \Lambda_{i}^{s}\right)$ is disjoint from $\mathrm{D} p_{j}\left(\mathrm{~T} \Lambda_{j}^{s}\right)$, for $i \neq j$. Then the images of the leaves $\Lambda_{i}^{s}$ under the map $p_{i}$ must transversely intersect the images of the leaves $\Lambda_{j}^{s}$ under the map $p_{j}$. Thus, the end points of their lifts in $\widetilde{F}$ are disjoint.

Second, suppose $\mathrm{D} p_{i}\left(\mathrm{~T} \Lambda_{i}^{s}\right)$ is injection for all $i$. If $\partial \widetilde{p}_{a_{1}}\left(\tilde{\Lambda}_{i}^{s}\right) \cap \partial \widetilde{p}_{a_{2}}\left(\tilde{\Lambda}_{i}^{s}\right) \neq \varnothing$, for some $a_{1}, a_{2} \in p_{i}^{-1}(y)$, then there exist leaves $\tilde{L}_{1}, \tilde{L}_{2} \subset \tilde{\Lambda}_{i}^{s}$ such that $\widetilde{p}_{a_{1}}\left(\tilde{L}_{1}\right)=$ $\tilde{p}_{a_{2}}\left(\tilde{L}_{2}\right)$. But this contradicts the injectiveness of $\mathrm{D} p_{i}\left(\mathrm{~T} \Lambda_{i}^{s}\right)$. So, we have finished the proof of one direction.

Suppose $\mathrm{D} p_{i}\left(\mathrm{~T} \Lambda_{i}^{s}\right)$ is not disjoint with $\mathrm{D} p_{j}\left(\mathrm{~T} \Lambda_{j}^{s}\right)$, ie there exist leaves $L \subset \Lambda_{i}^{s}$ and $J \subset \Lambda_{j}^{s}$ such that $\mathrm{D} p_{i}(L)=\mathrm{D} p_{j}(J)$. Then there exist a lift $\widetilde{L}$ of $L$ and a lift $\widetilde{J}$ of $J$ such that $\tilde{p}_{a}(\widetilde{L})=\widetilde{p}_{b}(\widetilde{J})$ for some $a \in p_{i}^{-1}(y)$ and some $b \in p_{j}^{-1}(y)$. This contradicts with the hypothesis of Theorem 3.3. The proof of the injectivity of the $\mathrm{D} p_{i}\left(\mathrm{~T} \Lambda_{i}^{s}\right)$ for all $i$ is similar.

\section{Applications}

The following theorem will be used to prove Corollary 4.3.

Theorem 4.1 (Farb and Mosher [4, Theorem 1.2]) Let $\pi_{1}(S)$ be the fundamental group of a surface $S$, and let $\Gamma_{\alpha}$ be the surface group extension of a group $G$. If $\Gamma_{\alpha}$ is word hyperbolic, then the homomorphism $\alpha: G \rightarrow \operatorname{MCG}(S)$ has finite kernel and convex cocompact image. 
Let $S \Gamma_{\varphi^{\boldsymbol{m}}}$ be a graph of surfaces with pseudo-Anosov regluings. Let $p: S_{e} \rightarrow F_{v}$ be an index $r$ covering map, where $F_{v}$ and $S_{e}$ are vertex and edge surfaces of $S \Gamma_{\varphi} \boldsymbol{m}$ respectively and $v$ is an end of $e$. Let $y \in F_{v}$ be a base point, let $\left\{x_{1}, \ldots, x_{r}\right\}=p^{-1}(y)$ denote the preimages of $y$ under the covering map $p$, and let $\tilde{x}_{i} \in \tilde{S}_{e}$ be a covering point of $x_{i}$ for $i \in\{1, \ldots, r\}$. Let $\tilde{p}_{i}:\left(\widetilde{S}_{e}, \tilde{x}_{i}\right) \rightarrow\left(\widetilde{F}_{v}, \tilde{y}\right)$ be a lift of $p$. Given a geodesic lamination $\Lambda \subset S_{e}$, if $\partial \tilde{p}_{i}(\tilde{\Lambda}) \bigcap \partial \tilde{p}_{j}(\tilde{\Lambda})=\varnothing$ for any $i \neq j \in\{1, \ldots, r\}$, we say that the ends of $p(\Lambda)$ are disjoint.

Lemma 4.2 Let $p: S_{e} \rightarrow F_{v}$ be a normal covering map. Let $\Phi \in \operatorname{MCG}\left(S_{e}\right)$ be a pseudo-Anosov mapping class, and let $\Lambda^{s}, \Lambda^{u}$ be the stable and unstable geodesic lamination respectively. Suppose the virtual centralizer of $\langle\Phi\rangle$ has trivial intersection with $G D_{p}$, the image of the deck transformation group of $p$ in $\operatorname{MCG}(S)$. Then the ends of both $p\left(\Lambda^{s}\right)$ and $p\left(\Lambda^{u}\right)$ are disjoint.

Proof We shall only prove that $\partial \widetilde{p}_{1}\left(\widetilde{\Lambda}^{s}\right)$ and $\partial \tilde{p}_{2}\left(\tilde{\Lambda}^{s}\right)$ are disjoint; a similar argument shows the pairwise disjointness of $\left\{\partial \widetilde{p}_{i}\left(\tilde{\Lambda}^{s}\right)\right\}$ and the pairwise disjointness of $\left\{\partial \tilde{p}_{i}\left(\widetilde{\Lambda}^{u}\right)\right\}$, for all $i \in\{1, \ldots, r\}$.

Let $D_{12}:\left(S_{e}, x_{1}\right) \rightarrow\left(S_{e}, x_{2}\right)$ be a deck transformation of the covering map $p$, and let $\widetilde{D}_{12}:\left(\widetilde{S}_{e}, \tilde{x}_{1}\right) \rightarrow\left(\widetilde{S}_{e}, \tilde{x}_{2}\right)$ be a lift of $D_{12}$.

Since $\tilde{p}_{1}=\tilde{p}_{2} \widetilde{D}_{12}, \tilde{p}_{1}\left(\widetilde{\Lambda}^{s}\right)=\tilde{p}_{2} \widetilde{D}_{12}\left(\tilde{\Lambda}^{s}\right)$. Hence, if the boundary points of the images of $\widetilde{\Lambda}^{s}$ under $\widetilde{p}_{1}$ and $\tilde{p}_{2}$ have one point in common, then $\widetilde{D}_{12}\left(\widetilde{\Lambda}^{s}\right)$ and $\widetilde{\Lambda}^{s}$ have one end point in common. Since $\widetilde{D}_{12}\left(\widetilde{\Lambda}^{s}\right)$ and $\tilde{\Lambda}^{s}$ are the lifts of the geodesic laminations $D_{12}\left(\Lambda^{s}\right)$ and $\Lambda^{s}$ respectively, by Fact 3, we know $D_{12}\left(\Lambda^{s}\right)=\Lambda^{s}$, where $D_{12}$ is considered as an element of $G D_{p} \subset \operatorname{MCG}(S)$. Applying [12, Theorem 3.5], if $D_{12}\left(\Lambda^{s}\right)=\Lambda^{s}$, then $D_{12}$ is contained in the virtual centralizer of $\langle\Phi\rangle$. This contradicts the hypothesis that the virtual centralizer of $\langle\Phi\rangle$ has trivial intersection with $G D_{p}$.

Corollary 4.3 Let $G, H$ be finite subgroups of $\operatorname{MCG}(S)$, and let $\Phi \in \operatorname{MCG}(S)$ be a pseudo-Anosov mapping class. If the virtual centralizer of $\langle\Phi\rangle$ has trivial intersection with $G$ and $H$, then for sufficiently large $M,\left\langle G, \Phi^{M} H \Phi^{-M}\right\rangle$ is a free product in $\operatorname{MCG}(S)$, ie $\left\langle G, \Phi^{M} H \Phi^{-M}\right\rangle \cong G * \Phi^{M} H \Phi^{-M}$, and its extension group is hyperbolic.

Remark If $G$ is a finite subgroup of $\operatorname{MCG}(S)$, then $G$ has a faithful representation as a subgroup of $\operatorname{Homeo}(S)$, which we continue to denote by $G$. The quotient $S / G$, called $F_{0}$, is a hyperbolic surface or orbifold. There exists a canonical embedding $i: \mathcal{P M} \mathcal{L}\left(F_{0}\right) \hookrightarrow \mathcal{P} \mathcal{M L}(S)$, where $\mathcal{P} \mathcal{M L}$ is the projective measured geodesic laminations space. Given a pseudo-Anosov mapping class $\Phi \in \operatorname{MCG}(S)$, if the stable 
and unstable geodesic laminations $\Lambda^{s}, \Lambda^{u} \notin i\left(\mathcal{P} \mathcal{M L}\left(F_{0}\right)\right)$, then the virtual centralizer of $\langle\Phi\rangle$ has trivial intersection with $G$. Therefore, it is easy to find a $\Phi \subset \operatorname{MCG}(S)$ which satisfies the hypothesis of this corollary.

Proof Let the symbols $G, H$ denote both the finite groups of $\operatorname{MCG}(S)$ and their faithful representations in $\operatorname{Homeo}(S)$. Let $F_{0}=S / G, F_{1}=S / H$. Let $p: S \rightarrow F_{0}$, $q: S \rightarrow F_{1}$ denote the corresponding covering maps, and let $p_{*}: \pi_{1}(S) \rightarrow \pi_{1}\left(F_{0}\right)$, $q_{*}: \pi_{1}(S) \rightarrow \pi_{1}\left(F_{1}\right)$ denote the induced maps on fundamental groups.

Let $G \Gamma$ be the following graph of groups:

$$
\pi_{1}\left(F_{0}\right) \stackrel{p_{*}}{\longleftarrow} \pi_{1}(S) \stackrel{\Phi^{M}}{\longrightarrow} \pi_{1}(S) \stackrel{q_{*}}{\longrightarrow} \pi_{1}\left(F_{1}\right)
$$

So, $\pi_{1}(G \Gamma)$ is the fundamental group of the following graph of surfaces $S \Gamma$ :

$$
F_{0} \stackrel{p}{\longleftarrow} S \stackrel{\phi^{M}}{\longrightarrow} S \stackrel{q}{\longrightarrow} F_{1}
$$

where $\phi \in \operatorname{Homeo}(S)$ is a pseudo-Anosov representative homeomorphism of $\Phi$.

There exists a short exact sequence

$$
1 \rightarrow \pi_{1}(S, x) \rightarrow \Gamma_{G * \Phi^{M} H \Phi^{-M}} \rightarrow G * \Phi^{M} H \Phi^{-M} \rightarrow 1 .
$$

It is not hard to see that $\Gamma_{G * \Phi^{M} H \Phi^{-M}}$ is isomorphic to $\Gamma_{G} *_{\pi_{1}(S)} \Gamma_{\Phi^{M} H \Phi^{-M}}$, and that $\Gamma_{G} *_{\pi_{1}(S)} \Gamma_{\Phi^{M}} H \Phi^{-M}$ is isomorphic to $\pi_{1}(G \Gamma)$.

By Theorem 4.1, if $\pi_{1}(G \Gamma)$ is a word hyperbolic group, then $\delta: G * \Phi^{M} H \Phi^{-M} \rightarrow$ $\operatorname{MCG}(S)$ has finite kernel. Since $G$ and $\Phi^{M} H \Phi^{-M}$ are finite groups, by applying [14, Theorem 3.11], a normal subgroup of $G * \Phi^{M} H \Phi^{-M}$ must be trivial or of finite index. Therefore, $\delta$ is an injection, which tells us that $\left\langle G, \Phi^{M} H \Phi^{M}\right\rangle \cong G * \Phi^{M} H \Phi^{-M}$.

In order to prove $\pi_{1}(G \Gamma)$ is word hyperbolic, we only need show that $S \Gamma$ is a hyperbolic graph of surfaces. By Lemma 4.2, we know that the ends of $p\left(\Lambda^{s}\right)$ and $q\left(\Lambda^{u}\right)$ are disjoint. Therefore, $S \Gamma$ is hyperbolic by Theorem 3.3.

Let $\mathcal{G}_{\phi^{m}}$ be as in Figure 3, where $S, F$ are genus 3 and 2 tori. Let $p: S \rightarrow F$ and $q: S \rightarrow F$ be covering maps and let $\phi$ be a pseudo-Anosov homeomorphism of the mapping class $\Phi$. Abusing of notation, we use $D_{p}, D_{q}$ for both the deck transformations of $p, q$ and the mapping classes of the deck transformations. It is easy to see that the deck transformation group $G D_{p}$ of $p$ contains only two elements, $D_{p}$ and the identity, the same is true for the deck transformation group of $q$. Further abusing of notation, we let $G D_{p}$ denote both the deck transformation group of $p$ and its image in $\operatorname{MCG}(S)$. 
Corollary 4.4 Suppose $a: S^{1} \rightarrow F$ and $c: S^{1} \rightarrow S$ are simple closed curves such that $p^{-1}\left(a\left(S^{1}\right)\right)=c\left(S^{1}\right), c\left(S^{1}\right) \subset q^{-1}\left(a\left(S^{1}\right)\right)$, and $q^{-1}\left(a\left(S^{1}\right)\right)$ is disconnected, as in Figure 4. In addition, suppose the virtual centralizer of $\langle\Phi\rangle$ has trivial intersection with the images of the deck transformation groups of $p$ and $q$ in $\operatorname{MCG}(S)$. Then $\pi_{1}\left(\mathcal{G}_{\phi^{m}}\right)$ is a hyperbolic group, when $m$ is sufficiently large.

Proof Let $z$ be the base point of $F$, let $x_{1}, x_{2}$ be the covering points of $z$ through the covering map $p$, and let $y_{1}, y_{2}$ be the covering points of $z$ through the covering map $q$. Let $\tilde{p}_{1}:\left(\widetilde{S}, \tilde{x}_{1}\right) \rightarrow(\widetilde{F}, \widetilde{z})$ and $\tilde{p}_{2}:\left(\widetilde{S}, \widetilde{x}_{2}\right) \rightarrow(\widetilde{F}, \widetilde{z})$ be the lifts of $p$, and let $\widetilde{D}_{p}:\left(\tilde{S}, \tilde{x}_{1}\right) \rightarrow\left(\tilde{S}, \tilde{x}_{2}\right)$ be the lift of $D_{p}$, and similarly for $q$.

According to Theorem 3.3 , we only need to show that $\left\{\partial \widetilde{p}_{1}\left(\widetilde{\Lambda}^{s}\right), \partial \widetilde{p}_{2}\left(\tilde{\Lambda}^{s}\right), \partial \widetilde{q}_{1}\left(\widetilde{\Lambda}^{u}\right)\right.$, $\left.\partial \widetilde{q}_{2}\left(\widetilde{\Lambda}^{u}\right)\right\}$ is a pairwise disjoint set.

First, using Lemma 4.2, we obtain $\partial \widetilde{p}_{1}\left(\widetilde{\Lambda}^{s}\right) \cap \partial \widetilde{p}_{2}\left(\widetilde{\Lambda}^{s}\right)=\varnothing, \partial \widetilde{q}_{1}\left(\widetilde{\Lambda}^{u}\right) \cap \partial \widetilde{q}_{2}\left(\widetilde{\Lambda}^{u}\right)=\varnothing$.

Second, we claim that if there exist $\partial \widetilde{p}_{r}\left(\widetilde{\Lambda}^{s}\right)$ and $\partial \widetilde{q}_{t}\left(\widetilde{\Lambda}^{u}\right)$ which are not disjoint, for some $r, t \in\{1,2\}$, then $p\left(\Lambda^{s}\right)=q\left(\Lambda^{u}\right)$ is a geodesic lamination on $F$. It follows that $\Lambda^{s}$ is a fixed point of $G D_{p} \subset \operatorname{MCG}(S)$. Therefore the virtual centralizer of $\langle\Phi\rangle$ and the deck transformation group have nontrivial intersection. A contradiction.

In the following, we will prove the above claim. Since $p_{*}\left(\pi_{1}(S)\right) \neq q_{*}\left(\pi_{1}(S)\right)$, and they are both index two subgroups of $\pi_{1}(F), p_{*}\left(\pi_{1}(S)\right) \cap q_{*}\left(\pi_{1}(S)\right)$ is an index four subgroup of $\pi_{1}(S)$. By calculating the Euler characteristic, we know there is a genus five surface $G$, and covering maps $i$ and $j$, such that the diagram in Figure 5 commutes, ie $p i=q j$.

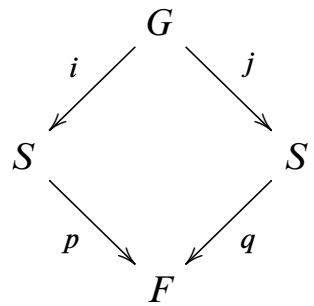

Figure 5

After straightening, the preimages of $i^{-1}\left(\Lambda^{s}\right)$ and $j^{-1}\left(\Lambda^{u}\right)$ are geodesic laminations, called $\mathcal{L}^{s}$ and $\mathcal{L}^{u}$, on $G$.

Without loss of generality, suppose $\widetilde{p_{1}}\left(\widetilde{\Lambda}^{s}\right)$ and $\widetilde{q_{1}}\left(\widetilde{\Lambda}^{u}\right)$ have one end point in common, then $\widetilde{p_{1}} \widetilde{i}\left(\widetilde{\mathcal{L}}^{s}\right)$ and $\widetilde{q_{1}} \widetilde{j}\left(\widetilde{\mathcal{L}}^{u}\right)$ have one end point in common. Combining this with the 
fact that $\tilde{p}_{1} \tilde{i}$ and $\tilde{q}_{1} \tilde{j}: \widetilde{G} \rightarrow \widetilde{F}$ are homeomorphisms, we know that $\widetilde{\mathcal{L}}^{s}$ and $\widetilde{\mathcal{L}}^{u}$ have one common end point. We claim that $\mathcal{L}^{s}$ and $\mathcal{L}^{u}$ are minimal geodesic laminations and fill the surface $G$. Therefore, if they have one common end point in the universal cover of $G$, then $\mathcal{L}^{s}=\mathcal{L}^{u}$. It is not hard to see that $\mathcal{L}^{s}$ is connected and without isolated leaves; therefore, according to [3, Corollary 4.7.2] $\mathcal{L}^{s}$ is minimal. The laminations $\mathcal{L}^{s}$ and $\mathcal{L}^{u}$ fill $G$ because they are lifts of filling laminations $\Lambda^{s}$ and $\Lambda^{u}$.

There exists some $m$ such that $\phi^{m}: S \rightarrow S$ is lifts by $i$ and $j$ to homeomorphisms of $G$. Denote the lift of $\phi^{m}: S \rightarrow S$ through $i$ as $\zeta: G \rightarrow G$, and the lift of $\phi^{-m}$ through $j$ as $\sigma: G \rightarrow G$. Notice that $\mathcal{L}^{s}$ is the stable geodesic lamination of $\zeta$ and $\mathcal{L}^{u}$ is the stable geodesic lamination of $\sigma$. Since $\mathcal{L}^{s}=\mathcal{L}^{u}$, there exist positive integers $k_{1}, k_{2}$, such that $\zeta^{k_{1}}$ is homotopic to $\sigma^{k_{2}}$.

Since $\zeta^{k_{1}}$ is homotopic to $\sigma^{k_{2}}$ and $p i=q j$, we know that $p i \zeta^{k_{1}}$ is homotopic to $q j \sigma^{k_{2}}$. Also, $p \phi^{k_{1} m_{i}}$ is homotopic to $q \phi^{-k_{2} m} j$, because $\phi^{k_{1} m_{i}} i=i \zeta^{k_{1}}$ and $\phi^{-k_{2} m} j=j \sigma^{k_{1}}$.

Let $p(c): S^{1} \rightarrow F$ be the closed curve which is the composition of $c: S^{1} \rightarrow S$ with the covering map $p: S \rightarrow F$. Similar notation are used for other compositions of closed curves with covering maps. The map $c^{2}: S^{1} \rightarrow S$ is defined to be the composition of the map $z \rightarrow z^{2}$ on the unit circle $S^{1}$ with map $c: S^{1} \rightarrow S$. Let $[a],[c]$ denote the conjugacy classes in the fundamental group of $F$ represented by the simple closed curve $a, c$.

Since $p(c)$ is homotopic to $a^{2}$ and $q(c)$ is homotopic to $a$, we see that $[a] \notin p_{*}\left(\pi_{1}(S)\right)$, $[a] \in q_{*}\left(\pi_{1}(S)\right)$, and $[a]^{2} \in p_{*}\left(\pi_{1}(S)\right) \cap q_{*}\left(\pi_{1}(S)\right)$. Hence there exists $\gamma: S^{1} \rightarrow G$ which is homotopic to a simple closed curve, such that $i(\gamma)$ is homotopic to $c$ and $j(\gamma)$ is homotopic to $c^{2}$. Therefore, $p \phi^{k_{1} m}(c), p \phi^{k_{1} m} i(\gamma), q \phi^{-k_{2} m} j(\gamma)$ and $q \phi^{-k_{2} m}\left(c^{2}\right)$ are homotopic to each other.

We claim that $q \phi^{-k_{2} m}(c)$ is homotopic to a simple closed curve on $F$. Let $\beta$ be the closed geodesic on $F$ which is homotopic to $q \phi^{-k_{2} m}(c)$. If $\beta$ is not simple, then there exits a point $z \in \beta\left(S^{1}\right)$, and a simple closed curve $\alpha: S^{1} \rightarrow S$ which is homotopic to $\phi^{-k_{2} m}(c)$, such that $q(\alpha)=\beta$, and there exists two points $x_{1} \neq x_{2} \in \alpha\left(S^{1}\right)$ such that $q\left(x_{1}\right)=q\left(x_{2}\right)=z$. Since $p \phi^{k_{1} m}(c)$ is homotopic to $q \phi^{-k_{2} m}\left(c^{2}\right)$, there exists a simple closed curve $\eta$ from $S^{1}$ to $S$ which is homotopic to $\phi^{k_{1} m}(c)$, and whose image under the map $p$ goes around $\beta$ twice, to be more precise, $p(\eta)=\beta^{2}$. It follows that there are four different points $y_{1}, y_{2}, y_{3}, y_{4} \in \eta\left(S^{1}\right)$ such that $p\left(y_{1}\right)=p\left(y_{2}\right)=$ $p\left(y_{3}\right)=p\left(y_{4}\right)=z$, which contradicts the fact that $p: S \rightarrow F$ is an index 2 covering map. 
By iterating, we have

$$
\begin{gathered}
p i \zeta^{n k_{1}} \text { is homotopic to } q j \sigma^{n k_{2}} \text { for all } n \in \mathbb{N}, \\
p \phi^{n k_{1} m} i(\gamma) \text { is homotopic to } q \phi^{-n k_{2} m} j(\gamma) \text { for all } n \in \mathbb{N}, \\
p \phi^{n k_{1} m}(c) \text { is homotopic to } q \phi^{-n k_{2} m}\left(c^{2}\right) \text { for all } n \in \mathbb{N} .
\end{gathered}
$$

By using the same argument, we know $q \phi^{-n k_{2} m}(c)$ is homotopic to a simple closed curve on $F$, for all $n \in \mathbb{N}$. Let $\alpha_{n}$ denote the geodesic in the free homotopy class of $\phi^{-n k_{2} m}(c)$. There exists a subsequence of $\alpha_{n}$, without loss of generality still call it $\alpha_{n}$, such that $\alpha_{n} \rightarrow \Lambda^{u}$ as $n \rightarrow \infty$. Since $q \phi^{-n k_{2} m}(c)$ is homotopic to a simple closed curve on $F$ for all n, the geodesics in the free homotopy classes of the $q \phi^{-n k_{2} m}(c)$ converge to a geodesic lamination $\Theta \subset F$, after passing to a subsequence. It follows that $q\left(\Lambda^{u}\right)$ is a geodesic lamination.

Notice that in the proof, we can only lift $\phi^{m}: S \rightarrow S$ by $i$ and $j$ to homeomorphisms of $G$ for some $m \in N$, but the end points of $\partial \widetilde{p}_{i}\left(\tilde{\Lambda}^{s}\right)$ and $\partial \widetilde{q}_{j}\left(\tilde{\Lambda}^{u}\right)$ for any $i, j \in\{1,2\}$ do not depend on $m$. Therefore, we have proved that $\left\{\partial \widetilde{p}_{1}\left(\widetilde{\Lambda}^{s}\right), \partial \widetilde{p}_{2}\left(\tilde{\Lambda}^{s}\right), \partial \widetilde{q}_{1}\left(\tilde{\Lambda}^{u}\right)\right.$, $\left.\partial \widetilde{q}_{2}\left(\tilde{\Lambda}^{u}\right)\right\}$ is a pairwise disjoint set. According to Theorem 3.3 , we know $\pi_{1}\left(\mathcal{G}_{\phi^{m}}\right)$ is hyperbolic for sufficiently large $m$.

\section{An example which is not abstractly commensurable to a surface-by-free group}

In this section, we will show that there exist a graph of surfaces whose fundamental group is hyperbolic, but which is not abstractly commensurable to any surface-byfree group, for any closed hyperbolic surface or orbifold $S^{\prime}$ and any free group $K$. Therefore, this group is different from all the groups constructed by Mosher [10]. By applying [5, Theorem 1.1], it follows that the example constructed here is not even quasi-isometric to any surface-by-free group.

Recall that, groups $G$ and $H$ are called abstractly commensurable, if there exist finite index subgroups $G_{1}<G$ and $H_{1}<H$, so that $G_{1}$ is isomorphic to $H_{1}$. A group $G$ is called a surface-by-free group, if there is a hyperbolic surface or a hyperbolic orbifold $S$, and a free group $K$, such that there exists a short exact sequence:

$$
1 \rightarrow \pi_{1}(S) \rightarrow G \rightarrow K \rightarrow 1
$$

First, we shall give a necessary and sufficient condition for a group to be abstractly commensurable to a surface-by-free group. Second, we shall construct a nonhyperbolic graph of surfaces $\mathcal{G}$, by applying the condition, whose fundamental group is not 
abstractly commensurable to any surface-by-free group. Finally, we shall construct a hyperbolic graph of surfaces $\mathcal{G}_{\phi^{m}}$ from $\mathcal{G}$ such that $\pi_{1}\left(\mathcal{G}_{\phi^{m}}\right)$ is not abstractly commensurable to any surface-by-free group.

Let $t$ denote the Bass-Serre tree of a graph of surfaces $S \Gamma$, and let $V, E$ denote the set of all the vertices and edges of $t$, respectively. The group $\pi_{1}(S \Gamma)$ acts on $t$ with subgroups $\operatorname{stab}(v)$ and $\operatorname{stab}(e)$, which stabilize the vertex $v \in V$ and the edge $e \in E$, respectively.

Lemma 5.1 The fundamental group of a graph of surfaces $S \Gamma$ is abstractly commensurable to a surface-by-free group if and only if $\left[\operatorname{stab}(v): \bigcap_{w \in V} \operatorname{stab}(w)\right]<\infty$, for all $v \in V$.

Proof According to Farb and Mosher [5], a finite index subgroup of a surface-by-free group is a surface-by-free group. If $\pi_{1}(S \Gamma)$ is abstractly commensurable to a surfaceby-free group, then there exists a finite index subgroup of $H$ of $\pi_{1}(S \Gamma)$ which is isomorphic to a surface-by-free group.

The group $H$ acts on $t$, a locally-finite tree with bounded valence, and the index $[\operatorname{stab}(v): H \cap \operatorname{stab}(v)] \leq\left[\pi_{1}(S \Gamma): H\right]$ is finite. $H$ acts on $t$ with compact quotient and $t$ may be identified with a Bass-Serre tree for $H$. Since $H$ is isomorphic to a surface-by-free group $\pi_{1}\left(S^{\prime}\right) \rtimes F$, where $S^{\prime}$ is a hyperbolic surface and $F$ is a finite rank free group, there exists a normal subgroup $N$ of $H$ which is isomorphic to $\pi_{1}\left(S^{\prime}\right)$ and so that $N$ acts trivially on $t$.

Let $N$ denote $\bigcap_{w \in V}(\operatorname{stab}(w) \cap H)$ which is a finite index subgroup of $\operatorname{stab}(v) \cap H$ for any vertex $v \in t$, ie $\left[\operatorname{stab}(v) \cap H: \bigcap_{w \in V}(\operatorname{stab}(w) \cap H)\right]<\infty$. Therefore,

$$
\begin{aligned}
{\left[\operatorname{stab}(v): \bigcap_{w \in V} \operatorname{stab}(w)\right] } & <\left[\operatorname{stab}(v): \bigcap_{w \in V}(\operatorname{stab}(w) \cap H)\right] \\
& =[\operatorname{stab}(v): H \cap \operatorname{stab}(v)]\left[H \cap \operatorname{stab}(v): \bigcap_{w \in V}(\operatorname{stab}(w) \cap H)\right] \\
& <\infty .
\end{aligned}
$$

This finishes the proof for one direction.

Now we will prove the other direction. The action of $\pi_{1}(S \Gamma)$ on $t$ induces a homomorphism $\sigma: \pi_{1}(S \Gamma) \rightarrow \operatorname{Aut}(t)$. Let $K=\bigcap_{w \in V} \operatorname{stab}(w), K=\operatorname{ker}(\sigma)$. Since $K$ is a finite index subgroup of $\operatorname{stab}(v)$ for any $v \in V, \pi_{1}(S \Gamma) / K$ acts on $t$ with finite edge and vertex stabilizers. In addition, $\pi_{1}(S \Gamma) / K$ acts on $t$ cocompactly. Therefore $t /\left(\pi_{1}(S \Gamma) / K\right)$ is a finite graph of finite groups. Applying [14, Theorem 7.3], it follows that $\pi_{1}(S \Gamma) / K$ is virtually free. Hence, $\pi_{1}(S \Gamma)$ is abstractly commensurable to a surface-by-free group. 
In the rest of this paper, let $\mathcal{G}$ denote a graph of surfaces as in Figure 6, where $S, F$, $p, q$ and the simple closed curves $c \subset S, a \subset F$ are as described in Corollary 4.4.

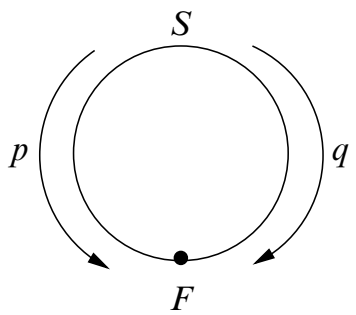

Figure 6

The conclusion of the next lemma that $\pi_{1}(\mathcal{G})$ is not commensurable to a surface-by-free group was discovered and proved independently by Chris Odden in his thesis, and also by Lee Mosher. I will give a different proof which will generalize to my later examples.

Define subgroups $L_{i}, R_{i}, G_{i}$ of $\pi_{1}(S)$, and subgroups $H_{i}$ of $\pi_{1}(F)$ by induction as follows:

- Let $H_{0}=\pi_{1}(F), G_{0}=\pi_{1}(S)$.

- Let $H_{1}=p_{*}\left(G_{0}\right) \cap q_{*}\left(G_{0}\right), L_{1}=p_{*}^{-1}\left(H_{1}\right), R_{1}=q_{*}^{-1}\left(H_{1}\right), G_{1}=L_{1} \cap R_{1}$.

- Let $H_{i+1}=p_{*}\left(G_{i}\right) \cap q_{*}\left(G_{i}\right), L_{i+1}=p_{*}^{-1}\left(H_{i+1}\right), R_{i+1}=q_{*}^{-1}\left(H_{i+1}\right)$, $G_{i+1}=L_{i+1} \cap R_{i+1}$.

From $p_{*}([c])=q_{*}\left(\left[c^{2}\right]\right)=\left[a^{2}\right]$, we know $\left[a^{2}\right] \in H_{1}$ but $[a] \notin H_{1},[c] \in L_{1},\left[c^{2}\right] \in R_{1}$ but $[c] \notin R_{1}$. Therefore, $L_{1} \neq R_{1},\left[c^{2}\right] \in G_{1},[c] \notin G_{1}$.

Similarly, from $p_{*}\left(\left[c^{2}\right]\right)=\left[a^{4}\right], q_{*}\left(\left[c^{2}\right]\right)=\left[a^{2}\right]$, we know $p_{*}\left(G_{1}\right) \neq q_{*}\left(G_{1}\right),\left[c^{2}\right] \in L_{2}$, $\left[c^{4}\right] \in R_{2}$, but $\left[c^{2}\right] \notin R_{2},\left[c^{4}\right] \in G_{2}$. Inductively, we have $\left[c^{2^{n}}\right] \in G_{n}, p_{*}\left(\left[c^{2^{n}}\right]\right)=\left[a^{4^{n}}\right]$, $q_{*}\left(\left[c^{2^{n}}\right]\right)=\left[a^{2^{n}}\right]$, so $p_{*}\left(G_{n}\right) \neq q_{*}\left(G_{n}\right),\left[c^{2^{n}}\right] \in L_{n+1},\left[c^{2^{n}}\right] \notin R_{n+1}$, but $\left[c^{2^{n+1}}\right] \in$ $R_{n+1}$. Hence, we get two sequences $\left\{L_{i}\right\}$ and $\left\{R_{i}\right\}$ of finite index normal subgroups of $\pi_{1}(S)$, the indexes of $\left[\pi_{1}(S): L_{i}\right]$ and $\left[\pi_{1}(S): R_{i}\right] \rightarrow \infty$ as $i \rightarrow \infty$.

Lemma 5.2 Suppose $\left[\pi_{1}(S): L_{i}\right]$ and $\left[\pi_{1}(S): R_{i}\right] \rightarrow \infty$ as $i \rightarrow \infty$, where $L_{i}, R_{i}$ are the sequences of finite index normal subgroups of $\pi_{1}(S)$ defined inductively above. If $L_{i} \neq R_{i}$ for all $i$, then $\pi_{1}(\mathcal{G})$ is not commensurable to a surface-by-free group.

Proof It is known that every edge or vertex stabilizer in the Bass-Serre tree $t$ is isomorphic to some edge or vertex group of the graph of groups. Let $e_{1}$ be an edge of the Bass-Serre tree $t$ such that the stabilizer $\operatorname{stab}\left(e_{1}\right)=\pi_{1}(S)$. Let $g$ be the 
generator of the underlying graph $\Gamma$ of the graph of spaces $\mathcal{G}$; by Scott and Wall [14] we know that if $\pi_{1}(S)$ is identified with $p_{*}\left(\pi_{1}(S)\right)$, then $q_{*}\left(\pi_{1}(S)\right)=g^{-1} \pi_{1}(S) g$. There exists a unique edge $e_{2} \in t$, such that $e_{2}=g e_{1}$. It is easy to see that $R_{1}=$ $q_{*}^{-1}\left(p_{*}\left(\pi_{1}(S) \cap q_{*}\left(\pi_{1}(S)\right)\right)=\operatorname{stab}\left(e_{1}\right) \cap \operatorname{stab}\left(e_{2}\right)\right.$. Let $e_{j}=g e_{j-1}$ for a positive integer $j$, let $\alpha_{i}$ be the oriented path $e_{1} * \cdots * e_{i}$ in the Bass-Serre tree $t$. Then $\bigcap_{\epsilon \in \alpha_{i}} \operatorname{stab}(\epsilon)=\bigcap_{j=1}^{i} \operatorname{stab}\left(e_{j}\right)=R_{i}$. Similarly, there exists another sequence of oriented paths $\left\{\beta_{k}\right\}$ in $t$ such that $\bigcap_{\epsilon \in \beta_{k}} \operatorname{stab}(\epsilon)=L_{k}$. Therefore, $\left[\pi_{1}(S): L_{i}\right] \rightarrow \infty$ and $\left[\pi_{1}(S): R_{i}\right] \rightarrow \infty$ imply $\left[\operatorname{stab}(e): \bigcap_{\epsilon \in E} \operatorname{stab}(\epsilon)\right]=\infty$. For the case studied here, every edge stabilizer is a finite index subgroup of some vertex stabilizer where the vertex is an end point of that edge. So, $\left[\operatorname{stab}(e): \bigcap_{\epsilon \in E} \operatorname{stab}(\epsilon)\right]=\infty$ implies $\left[\operatorname{stab}(v): \bigcap_{w \in V} \operatorname{stab}(w)\right]=\infty$. According to Lemma 5.1, $\pi_{1}(\mathcal{G})$ is not commensurable to a surface-by-free group.

In order to construct a group which is not abstractly commensurable to a surface-by-free group, a first strategy might be to find a pseudo-Anosov mapping class $\Phi$ which fixes all the finite index normal subgroups of $\pi_{1}(S)$. But unfortunately, the theorem below tells us that there does not exist such a pseudo-Anosov mapping class.

Theorem 5.3 Let $S_{n}$ be a closed surface of genus $n$, where $n \geq 2$. For any $\Phi \in$ $\operatorname{Aut}\left(\pi_{1}\left(S_{n}\right)\right)$, if $\Phi$ fixes all the finite index normal subgroups of $\pi_{1}\left(S_{n}\right)$, then $\Phi \in$ $\operatorname{Inn}\left(\pi_{1}\left(S_{n}\right)\right)$.

Before proving this theorem, we introduce some related history and preliminaries first. Lubotzky [9] proved that for any free group $F_{n}, n \geq 2$, if $\Psi \in \operatorname{Aut}\left(F_{n}\right)$ fixes all the finite index normal subgroups of $F_{n}$, then $\Psi \in \operatorname{Inn}\left(F_{n}\right)$. In particular, every normal automorphism of $F_{n}$ is inner. Bogopolski, Kudryavtseva and Zieschang [2] proved that for any closed hyperbolic surface $S_{n}$ of genus $n$ not less than 2 , if $\Phi \in \operatorname{Aut}\left(\pi_{1}\left(S_{n}\right)\right)$ fixes all the normal subgroups (not necessarily of finite index) of $\pi_{1}\left(S_{n}\right)$, then $\Phi \in$ $\operatorname{Inn}\left(\pi_{1}\left(S_{n}\right)\right)$. The main theorem in that paper says for any nonseparating simple closed curve $\alpha$ on $S$, up to conjugate equivalence, $\alpha$ is the only nonseparating simple closed curve in its normal closure. The theorem in [2] is the following:

Theorem 5.4 (Bogopolski, Kudryavtseva and Zieschang [2]) Let $S$ be a closed orientable surface and $g, h$ are nontrivial elements of $\pi_{1}(S)$ both containing simple closed two-sided curves $\gamma$ and $\kappa$, resp. The group element $h$ belongs to the normal closure of $g$ if and only if $h$ is conjugate to $g^{\epsilon}$ or to $\left(g u g^{-1} u^{-1}\right)^{\epsilon}, \epsilon \in\{1,-1\}$; here $u$ is a homotopy class containing a simple closed curve $\mu$ which properly intersects $\gamma$ exactly once.

I would like to thank Jason Deblois for help with Lemma 5.5. 
Lemma 5.5 For any two nontrivial, non-freely homotopic, nonseparating simple closed curves $a$ and $b$ on $S$, let $[a],[b]$ denote their homotopy classes in $\pi_{1}(S)$. Then there exists a finite index normal subgroup $N \in \pi_{1}(S)$, such that $[a] \in N$ and $[b] \notin N$.

A group $G$ is said to be residually finite, if for any element $g \in G, g \neq 1$, there exists a finite group $K$ and a homomorphism $h: G \rightarrow K$, such that $h(g) \neq 1$.

A Haken manifold is a compact, orientable, irreducible 3-manifold which contains a 2-sided incompressible surface.

Proof Let $M=S \times I$, where $I$ is the interval $[0,1] . \pi_{1}(M)$ is isomorphic to $\pi_{1}(S)$. Since $a$ is a simple closed curve on $S$, attach a 2-handle $B$ to $M$ along $a \times\{0\} \cup a \times\{1\}$ obtaining a Haken manifold $M^{\prime}$. This attachment gives a surjective homomorphism $\epsilon: \pi_{1}(M) \rightarrow \pi_{1}\left(M^{\prime}\right)$, and the kernel is the normal closure of $[a]$. Since $a$ is the only nonseparating simple closed curve in the normal closure of $[a]$, by applying Theorem 5.4, it follows that $[b]$ does not belong to the kernel of $\epsilon$.

According to [7, Theorem 1.1], $\pi_{1}\left(M^{\prime}\right)$ is residually finite. So, for $[b] \in \pi_{1}(M)$, there exists a finite group $K$ and a homomorphism $\delta: \pi_{1}\left(M^{\prime}\right) \rightarrow K$ such that $[b] \notin \operatorname{ker}(\delta)$. Let $N$ denote the kernel $\operatorname{ker}(\delta \circ \epsilon)$. Obviously, $N$ is a finite index normal subgroup of $\pi_{1}(S)$, and $[a] \in N$, but $[b] \notin N$.

Proof of Theorem 5.3 Let $\Phi$ be an element of $\operatorname{Aut}\left(\pi_{1}(S)\right)$, and let $\phi$ be a representative of it in $\operatorname{Homeo}(S)$. According to [2], if $\Phi \notin \operatorname{Inn}\left(\pi_{1}(S)\right)$, then there exists a nonseparating simple closed curve $a$ on $S$ such that $a$ and $\phi(a)$ are not freely homotopic to each other. By Lemma 5.5, there exists a finite index normal subgroup $N \triangleleft \pi_{1}(S)$, such that $[a] \in N$ and $[\phi(a)] \notin N$. It follows that $\Phi(N) \neq N$.

In the following, we shall construct a pseudo-Anosov mapping class which does not fix all the finite index normal subgroups of $\pi_{1}(S)$, but fixes $L_{i}$ and $R_{i}$ as in Lemma 5.2. Also, let $\mathcal{G}_{\phi^{m}}$ be a graph of surfaces as in Figure 3, where $F, S, p, q$ as described in Corollary 4.4.

Theorem 5.6 There exists a pseudo-Anosov homeomorphism $\phi \in \operatorname{Homeo}(S)$, so that $\pi_{1}\left(\mathcal{G}_{\phi^{m}}\right)$ is hyperbolic but is not commensurable to a surface-by-free group.

Proof If there exists a pseudo-Anosov homeomorphism $\phi$, such that $\phi_{*}\left(L_{i}\right)=L_{i}$ and $\phi_{*}\left(R_{i}\right)=R_{i}$, then $\left[\operatorname{stab}(e): \bigcap_{\epsilon \in E} \operatorname{stab}(\epsilon)\right]=\infty$, according to Lemma 5.2. Therefore, $\pi_{1}\left(\mathcal{G}_{\phi^{m}}\right)$ is not commensurable to a surface-by-free group. 


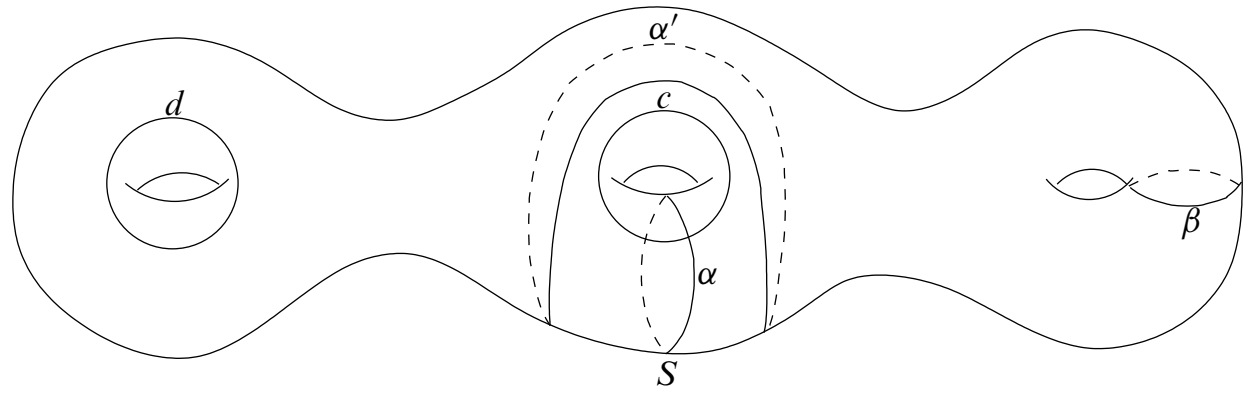

Figure 7

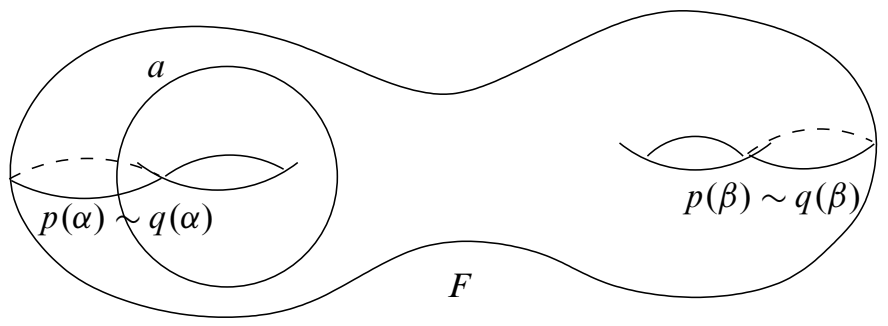

Figure 8

The curves mentioned in this theorem are shown in Figure 7 and Figure 8, which are a refinement of Figure 4.

First, we will describe the covering maps $p$ and $q$ in more detail. Let $p^{-1}\left(a^{2}\right)=c$, $q^{-1}(a)=c \cup d$. It is easy to see that $p(\alpha)$ is homotopic to $q(\alpha) \subset F$ and $p(\beta)$ is homotopic to $q(\beta) \subset F$, where $\alpha, \beta \subset S$ are as in Figure 7. Therefore $[\alpha],[\beta] \in L_{i} \cap R_{i}$ for all $i$.

Second, we claim that if $\gamma$ is a simple closed curve in $S$, such that $[\gamma] \in L_{i}$ for some $i$, then the induced map $\left(\tau_{\gamma}\right)_{*}$ of the Dehn twist $\tau_{\gamma}$ fixes $L_{i}$. Let $S^{\prime}$ be the cover of $S$ corresponding to $L_{i}$, and let $\Gamma$ be the finite set of preimages of $\gamma$ to $S^{\prime}$. Then $\left(\tau_{\Gamma}\right)_{*}=\left.\left(\tau_{\gamma}\right)_{*}\right|_{L_{i}}$, where $\tau_{\Gamma}: S^{\prime} \rightarrow S^{\prime}$.

If we can find disjointly essential curve systems $\mathcal{C}$ and $\mathcal{D}$ which satisfy the conditions in Theorem 2.1 and if all the homotopy classes of the elements of $\mathcal{C}$ and $\mathcal{D}$ belong to $L_{i}$ and $R_{i}$ for all $i$, then we can construct a pseudo-Anosov homeomorphism $\phi$ as described in Theorem 2.1, such that $\phi_{*}$ fixes $L_{i}$ and $R_{i}$ for all $i$.

In the following, we will prove that there exist disjointly essential curve systems $\mathcal{C}=\alpha \cup \hat{\alpha}$, and $\mathcal{D}=\beta \cup \widehat{\beta}$, such that $\mathcal{C} \cup \mathcal{D}$ fills $S$, where $\alpha, \beta$ as in Figure 7. In addition, $[\alpha],[\hat{\alpha}],[\beta]$ and $[\widehat{\beta}] \in \bigcap_{i}\left(L_{i} \cap R_{i}\right)$. 
In order to find a simple closed curve $\widehat{\alpha}$ satisfying the above conditions, first, we will show that there exists a simple closed curve $\alpha^{\prime}$ such that $\left[\alpha^{\prime}\right] \in \bigcap_{i}\left(L_{i} \cap R_{i}\right)$. Since $L_{i}$ and $R_{i}$ are finite index normal subgroups of $\pi_{1}(S)$ and $[\alpha] \in \bigcap_{i}\left(L_{i} \cap R_{i}\right)$, the normal closure $N_{\alpha}$ of $[\alpha]$ is a subgroup of $\bigcap_{i}\left(L_{i} \cap R_{i}\right)$. Recall that the normal closure $N_{\alpha}$ of $[\alpha]$ is the smallest normal subgroup of $\pi_{1}(S)$ which contains $[\alpha]$. Applying Theorem 5.4 , we only need the easy direction of this theorem, the separating curve $\alpha^{\prime}$ as in Figure 7 represents an element in $N_{\alpha}$.

Second, we shall construct a simple closed curve $\hat{\alpha}$ on $S$ from the simple closed curve $\alpha^{\prime}$. From Mosher [11], we know there exists a short exact sequence

$$
1 \rightarrow\left\langle T_{\alpha}\right\rangle \rightarrow \operatorname{stab}(\alpha) \rightarrow \operatorname{MCG}(S-\alpha) \rightarrow 1
$$

where $\left\langle T_{\alpha}\right\rangle$ is the cyclic subgroup of $\operatorname{MCG}(S)$ generated by the mapping class $T_{\alpha}$ of the Dehn twist $\tau_{\alpha}$ around $\alpha, \operatorname{stab}(\alpha)$ is a subgroup of $\operatorname{MCG}(S)$ which fixes $\alpha, S-\alpha$ is a surface obtained by cutting $S$ along $\alpha$. The homomorphism $\iota$ : $\operatorname{stab}(\alpha) \rightarrow \operatorname{MCG}(S-\alpha)$ is defined by $\left.\Phi \rightarrow \Phi\right|_{S-\alpha}$, for $\Phi \in \operatorname{stab}(\alpha)$.

Choose a pseudo-Anosov homeomorphism $\psi \in \operatorname{Homeo}(S-\alpha)$. Pass to a high enough power of $\psi$, so that $\hat{\alpha}=\psi\left(\alpha^{\prime}\right)$ is very close to the stable geodesic lamination $\Lambda_{\psi}^{S}$ of $\psi$; therefore, $\hat{\alpha} \cup \beta$ fills $S-\alpha$. Also, $\hat{\alpha}$ is disjoint from $\alpha$ because $\alpha^{\prime}$ is disjoint from $\alpha$. Using the same method, we can find a simple closed curve $\hat{\beta}$ which is disjoint with $\beta$ and $\widehat{\beta} \cup \alpha$ fills $S-\beta$.

Let $\mathcal{C}=\{\alpha, \widehat{\alpha}\}, \mathcal{D}=\{\beta, \hat{\beta}\}$, it is easy to see that $\mathcal{C} \cup \mathcal{D}$ fills $S$. According to Theorem 2.1, if $\phi_{0}$ is a homeomorphism of $S$, such that $\tau_{\alpha}^{+}, \tau_{\hat{\alpha}}^{+}, \tau_{\beta}^{-}$and $\tau_{\hat{\beta}}^{-}$appear at least once in $\phi_{0}$, then $\phi_{0}$ is a pseudo-Anosov homeomorphism. Since $[\alpha],[\hat{\alpha}],[\beta]$, $[\widehat{\beta}] \in \bigcap_{i}\left(L_{i} \cap R_{i}\right)$, we have that $\left(\phi_{0}\right)_{*}$ fixes $L_{i}$ and $R_{i}$ for all $\mathrm{i}$.

In order to finish the proof of this theorem, according to Corollary 4.4, we only need to show that there exists some pseudo-Anosov homeomorphism $\phi$ constructed as above, so that the virtual centralizer $\operatorname{VC}\langle\Phi\rangle$ of $\langle\Phi\rangle$ has trivial intersection with the mapping classes of the deck transformation groups of the covering maps $p$ and $q$, respectively, where $\Phi \in \operatorname{MCG}(S)$ is the mapping class of $\phi$. Abusing notation, denote both the deck transformations and the mapping classes of the deck transformations by $D_{p}$ and $D_{q}$. The deck transformation group of $p$ has only two elements $D_{p}$ and the identity.

Let $\phi_{0}$ be a pseudo-Anosov homeomorphism of $S$ constructed above, and let $\Phi_{0}$ be its mapping class. Let $\Lambda_{\phi_{0}}^{s}$ and $\Lambda_{\phi_{0}}^{u}$ be the stable and unstable geodesic laminations of $\phi_{0}$, respectively. It is known that $\Phi_{0}$ fixes $L_{i}$ and $R_{i}$ for all $i$.

Suppose the deck transformation group of $p$ has nontrivial intersection with the virtual centralizer of $\left\langle\Phi_{0}\right\rangle$, ie $D_{p}\left(\Lambda_{\phi_{0}}^{s}\right)=\Lambda_{\phi_{0}}^{s}$. We claim that $D_{p}\left(T_{\alpha}\left(\Lambda_{\phi_{0}}^{s}\right)\right) \neq T_{\alpha}\left(\Lambda_{\phi_{0}}^{s}\right)$, 
where $T_{\alpha}$ is the mapping class of the Dehn twist $\tau_{\alpha}$. Notice that $T_{\alpha}\left(\Lambda_{\phi_{0}}^{s}\right)$ is the stable geodesic lamination of the pseudo-Anosov mapping class $T_{\alpha} \Phi_{0} T_{\alpha}^{-1}$, and that $T_{\alpha} \Phi_{0} T_{\alpha}^{-1}$ fixes $L_{i}$ and $R_{i}$ for all $i$. If the claim is true and letting $\Phi_{1}=T_{\alpha} \Phi_{0} T_{\alpha}^{-1}$, then $\operatorname{VC}\left\langle\Phi_{1}\right\rangle$ has trivial intersection with $D_{p}$.

We shall prove the claim. Notice that there exists a simple closed curve $\gamma$ on $S$, which is disjoint from $\alpha$, such that $D_{p}(\alpha)=\gamma$. According to [8, Lemma 4.1.C], $D_{p} T_{\alpha} D_{p}^{-1}=T_{D_{p}(\alpha)}=T_{\gamma}$.

Suppose $D_{p} T_{\alpha}\left(\Lambda_{\phi_{0}}^{s}\right)=T_{\alpha}\left(\Lambda_{\phi_{0}}^{s}\right)$, then

$$
D_{p} T_{\alpha}\left(\Lambda_{\phi_{0}}^{s}\right)=T_{\gamma} D_{p}\left(\Lambda_{\phi_{0}}^{s}\right)=T_{\gamma}\left(\Lambda_{\phi_{0}}^{s}\right) .
$$

Therefore, $T_{\alpha}\left(\Lambda_{\phi_{0}}^{s}\right)=T_{\gamma}\left(\Lambda_{\phi_{0}}^{s}\right)$. It follows that $T_{\alpha}^{-1} T_{\gamma} \in \mathrm{VC}\left\langle\Phi_{0}\right\rangle$, but from [12, Theorem 3.5], we know that $\operatorname{VC}\left\langle\Phi_{0}\right\rangle$ has $\left\langle\Phi_{0}\right\rangle$ as a finite index subgroup. Hence, up to some power $m,\left(T_{\alpha}^{-1} T_{\gamma}\right)^{m} \in\left\langle\Phi_{0}\right\rangle$, but obviously $\left(T_{\alpha}^{-1} T_{\gamma}\right)^{m}$ is neither pseudo-Anosov nor the identity, so it is not an element of $\left\langle\Phi_{0}\right\rangle$. Therefore $D_{p} T_{\alpha}\left(\Lambda_{\phi_{0}}^{s}\right) \neq T_{\alpha}\left(\Lambda_{\phi_{0}}^{s}\right)$. If, in addition, $D_{q} T_{\alpha}\left(\Lambda_{\phi_{0}}^{u}\right) \neq T_{\alpha}\left(\Lambda_{\phi_{0}}^{u}\right)$, then take $\Phi=\Phi_{1}$ and the theorem is proved in this case.

If $D_{q} T_{\alpha}\left(\Lambda_{\phi_{0}}^{u}\right)=T_{\alpha}\left(\Lambda_{\phi_{0}}^{u}\right)$, then we claim $D_{q} T_{\alpha}^{2}\left(\Lambda_{\phi_{0}}^{u}\right) \neq T_{\alpha}^{2}\left(\Lambda_{\phi_{0}}^{u}\right)$. If the claim is not true, then

$$
\begin{aligned}
D_{q} T_{\alpha}^{2}\left(\Lambda_{\phi_{0}}^{u}\right) & =T_{\alpha}^{2}\left(\Lambda_{\phi_{0}}^{u}\right) \\
& =T_{\alpha}\left(D_{q} T_{\alpha}\left(\Lambda_{\phi_{0}}^{u}\right)\right) \\
& =D_{q} T_{\theta}\left(T_{\alpha}\left(\Lambda_{\phi_{0}}^{u}\right)\right),
\end{aligned}
$$

where $\theta=D_{q}(\alpha)$ is a simple closed curve on $S$ disjoint from $\alpha$. Therefore,

$$
T_{\alpha}^{-1} T_{\theta}^{-1} D_{q}^{-1} D_{q} T_{\alpha}^{2}\left(\Lambda_{\phi_{0}}^{u}\right)=\Lambda_{\phi_{0}}^{u} .
$$

It follows that $T_{\alpha}^{-1} T_{\theta}^{-1} T_{\alpha}^{2}\left(\Lambda_{\phi_{0}}^{u}\right)=\Lambda_{\phi_{0}}^{u}$. Since $\theta, \alpha$ are disjoint simple closed curves, we have $T_{\alpha} T_{\theta}^{-1}=T_{\theta}^{-1} T_{\alpha}$. Therefore, $T_{\alpha}^{-1} T_{\theta}^{-1} T_{\alpha}^{2}\left(\Lambda_{\phi_{0}}^{u}\right)=T_{\theta}^{-1} T_{\alpha}\left(\Lambda_{\phi_{0}}^{u}\right)=\Lambda_{\phi_{0}}^{u}$. By the same reason as in the above argument, it is impossible.

Replacing $T_{\alpha}, T_{\gamma}$ by $T_{\alpha}^{2}, T_{\gamma}^{2}$ in the above proof of $D_{p} T_{\alpha}\left(\Lambda_{\phi_{0}}^{s}\right) \neq T_{\alpha}\left(\Lambda_{\phi_{0}}^{s}\right)$, we see that $D_{p} T_{\alpha}^{2}\left(\Lambda_{\phi_{0}}^{s}\right) \neq T_{\alpha}^{2}\left(\Lambda_{\phi_{0}}^{s}\right)$. Taking $\Phi=T_{\alpha}^{2} \Phi_{0} T_{\alpha}^{-2}$, the theorem is proved.

\section{References}

[1] M Bestvina, M Feighn, A combination theorem for negatively curved groups, J. Differential Geom. 35 (1992) 85-101 MR1152226 
[2] O Bogopolski, E Kudryavtseva, H Zieschang, Simple curves on surfaces and an analog of a theorem of Magnus for surface groups, Math. Z. 247 (2004) 595-609 MR2114430

[3] A J Casson, S A Bleiler, Automorphisms of surfaces after Nielsen and Thurston, London Math. Soc. Student Texts 9, Cambridge Univ. Press (1988) MR964685

[4] B Farb, L Mosher, Convex cocompact subgroups of mapping class groups, Geom. Topol. 6 (2002) 91-152 MR1914566

[5] B Farb, L Mosher, The geometry of surface-by-free groups, Geom. Funct. Anal. 12 (2002) 915-963 MR1937831

[6] A Fathi, F Laudenbach, V Poenaru, editors, Travaux de Thurston sur les surfaces, Astérisque 66-67, Soc. Math. France, Paris (1979) MR568308 Séminaire Orsay, With an English summary

[7] J Hempel, Residual finiteness of surface groups, Proc. Amer. Math. Soc. 32 (1972) 323 MR0295352

[8] N V Ivanov, Mapping class groups, from: "Handbook of geometric topology", (R J Daverman, R B Sher, editors), North-Holland, Amsterdam (2002) 523-633 MR1886678

[9] A Lubotzky, Normal automorphisms of free groups, J. Algebra 63 (1980) 494-498 MR570726

[10] L Mosher, A hyperbolic-by-hyperbolic hyperbolic group, Proc. Amer. Math. Soc. 125 (1997) 3447-3455 MR1443845

[11] L Mosher, Train track expansions of measured foliations, Preprint (2003) Available at http://andromeda.rutgers.edu/ mosher/arationality_03_02_15.pdf

[12] L Mosher, Geometric survey of subgroups of mapping class groups, from: "Handbook of Teichmüller theory. Vol. I", (A Papadopoulos, editor), IRMA Lect. Math. Theor. Phys. 11, Eur. Math. Soc., Zürich (2007) 387-410 MR2349675

[13] R C Penner, A construction of pseudo-Anosov homeomorphisms, Trans. Amer. Math. Soc. 310 (1988) 179-197 MR930079

[14] P Scott, C T C Wall, Topological methods in group theory, from: "Homological group theory (Proc. Sympos., Durham, 1977)”, (C T C Wall, editor), London Math. Soc. Lecture Note Ser. 36, Cambridge Univ. Press (1979) 137-203 MR564422

Department of Mathematics, The Ohio State University

231 West 18th Avenue, Columbus OH 43210, USA

min@math.ohio-state.edu

Received: 14 August 2009 Revised: 14 December 2009 